\title{
Search for trans-iron elements in hot, helium-rich white dwarfs with the HST Cosmic Origins Spectrograph ${ }^{\star, \star \star}$
}

\author{
D. Hoyer ${ }^{1}$, T. Rauch ${ }^{1}$, K. Werner ${ }^{1}$, and J.W. Kruk ${ }^{2}$ \\ ${ }^{1}$ Institute for Astronomy and Astrophysics, Kepler Center for Astro and Particle Physics, Eberhard Karls University, Sand 1, 72076 \\ Tübingen, Germany \\ e-mail: werner@astro.uni-tuebingen.de \\ 2 NASA Goddard Space Flight Center, Greenbelt, MD 20771, USA
}

Received 2 December 2017 / Accepted 3 January 2018

\begin{abstract}
The metal abundances in the atmospheres of hot white dwarfs (WDs) entering the cooling sequence are determined by the preceding Asymptotic Giant Branch (AGB) evolutionary phase and, subsequently, by the onset of gravitational settling and radiative levitation. In this paper, we investigate three hot He-rich WDs, which are believed to result from a late He-shell flash. During such a flash, the He-rich intershell matter is dredged up and dominates the surface chemistry. Hence, in contrast to the usual H-rich WDs, their spectra allow direct access to s-process element abundances in the intershell that were synthesized during the AGB stage. In order to look for trans-iron group elements (atomic number $Z>29$ ), we performed a non-local thermodynamic equilibrium model atmosphere analysis of new ultraviolet spectra taken with the Cosmic Origins Spectrograph aboard the Hubble Space Telescope. One of our program stars is of PG 1159 spectral type; this star, PG 1707+427, has effective temperature $T_{\text {eff }}=85000 \mathrm{~K}$, and surface gravity $\log g=7.5$. The two other stars are DO white dwarfs: WD $0111+002$ has $T_{\text {eff }}=58000 \mathrm{~K}$ and $\log g=7.7$, and PG 0109+111 has $T_{\text {eff }}=70000 \mathrm{~K}$ and $\log g$ $=8.0$. These stars trace the onset of element diffusion during early WD evolution. While zinc is the only trans-iron element we could detect in the PG 1159 star, both DOs exhibit lines from Zn, Ga, Ge, Se; one additionally exhibits lines from Sr, Sn, Te, and I and the other from As. Generally, the trans-iron elements are very abundant in the DOs, meaning that radiative levitation must be acting. Most extreme is the almost six orders of magnitude oversolar abundance of tellurium in PG 0109+111. In terms of mass fraction, it is the most abundant metal in the atmosphere. The two DOs join the hitherto unique hot DO RE 0503-289, in which 14 trans-iron elements had even been identified.
\end{abstract}

Key words. stars: abundances - stars: atmospheres - white dwarfs - stars: evolution - atomic data

\section{Introduction}

The hot white dwarf RE0503-289 is unique because a large number of trans-iron elements were discovered in its photosphere (Werner et al. 2012b). Abundance analyses of 14 species with atomic numbers in the range $Z=30-56$, i.e., zinc through barium, revealed high quantities of up to about $10^{4}$ times the solar values (Rauch et al. 2017b, and references therein).

RE 0503-289 is a non-DA white dwarf (WD) with a heliumdominated atmosphere (spectral type hot DO, effective temperature $T_{\text {eff }}=70000 \pm 2000 \mathrm{~K}$, surface gravity $\log g=7.5 \pm$ 0.1; Rauch et al. 2016b). We concluded that the high heavymetal abundances are probably caused by radiative levitation (Rauch et al. 2016a). If true, then we should find the same phenomenon among other hot DOs with similar temperature and gravity. Therefore we performed ultraviolet (UV) spectroscopy of three other WDs with the Cosmic Origins Spectrograph (COS) aboard the Hubble Space Telescope (HST). Two of these WDs (PG 0109+111 and WD 0111+002, alias HS 0111+0012) are also of the hot DO spectral type. The third (PG 1707+427) was

\footnotetext{
* Based on observations with the NASA/ESA Hubble Space Telescope, obtained at the Space Telescope Science Institute, which is operated by the Association of Universities for Research in Astronomy, Inc., under NASA contract NAS5-26666.

$\star \star$ Based on observations made with the NASA-CNES-CSA Far Ultraviolet Spectroscopic Explorer.
}

drawn from the PG 1159-type class, which are hot, non-DA (pre-) WDs with large amounts of atmospheric carbon and oxygen. Their surface chemistry is the result of a late He-shell flash that dredged up the intershell matter located between the $\mathrm{H}$ and He burning shells of the previous Asymptotic Giant Branch (AGB) evolutionary phase (Werner \& Herwig 2006). PG 1159 stars without remaining hydrogen evolve into DO WDs as soon as gravitational settling starts to remove these species from the atmosphere. The choice of a PG 1159-type target was motivated by the fact that the trans-iron element abundances are not yet affected by diffusion and, hence, should reflect the abundances in the intershell layer created by the s-process during the AGB phase.

This paper is structured as follows. First, we introduce our program stars in Sect.2, and proceed with a description of our new and archival observations (Sect.3). We then present our spectral analysis to determine the basic atmospheric parameters $T_{\text {eff }}, \log g$, and metal abundances (Sect. 4). We discuss our results and conclude in Sect. 5.

\section{The program stars}

For our new observations we chose two hot DOs and a PG1159 star, which are bright enough to obtain UV spectra with a good signal-to-noise ratio enabling the identification of even weak metal lines. The location of the program stars in the 
Table 1. Observation log of our three program stars.

\begin{tabular}{ccccccccc}
\hline \hline Star & Instrument & Dataset & Grating & $R$ & $\lambda / \AA$ & $t_{\exp } / \mathrm{s}$ & Date & PI \\
\hline \multirow{2}{*}{ WD 0111+002 } & HST/COS & LCK201010 & \multirow{2}{*}{ G130M } & $13000-19000$ & $1070-1210,1224-1365$ & 8017 & $2015-11-17$ & Werner \\
& HST/GHRS & Z3GM0304T & G160M & 18000 & $1228-1264$ & 4570 & $1996-11-11$ & Werner \\
& FUSE & A0130301000 & & 20000 & $915-1188$ & 5515 & $2001-08-07$ & Dreizler \\
PG 0109+111 & HST/COS & LCK202010 & G130M & $13000-19000$ & $1070-1210,1224-1365$ & 8005 & $2015-12-01$ & Werner \\
& FUSE & A0130202000 & & 20000 & $915-1188$ & 4355 & $2000-11-23$ & Dreizler \\
PG 1707+427 & HST/COS & LCK203010 & G130M & $13000-19000$ & $1070-1210,1224-1365$ & 14344 & $2015-09-14$ & Werner \\
& HST/GHRS & Z2T20304T & G140L & $1800-2200$ & $1165-1460$ & 1197 & $1995-08-06$ & Heber \\
& FUSE & P1320401000 & & 20000 & $915-1188$ & 14541 & $2000-06-06$ & Kruk \\
\hline
\end{tabular}

Notes. For the HST/COS observations the two wavelength intervals covered by detector segments A and B are given. All FUSE spectra were obtained with the LWRS aperture. Resolving power is $R$. Exposure time is $t_{\text {exp }}$.

$g-T_{\text {eff }}$ diagram (already with our improved parameters for PG 0109+111) together with other hydrogen-deficient stars is shown in Fig. 1.

\subsection{WD 0111+002 and PG0109+111 (DO WDs)}

The non-local thermodynamic equilibrium (NLTE) analysis of optical spectroscopy yielded $T_{\text {eff }}=65000 \pm 5000 \mathrm{~K}, \log g=$ $7.8 \pm 0.3$ for WD $0111+002$ and $T_{\text {eff }}=110000 \pm 10000 \mathrm{~K}$, $\log g=8.0 \pm 0.3$ for PG0109+111. A carbon abundance of $\mathrm{C}=0.003$ in both stars was derived (Dreizler \& Werner 1996); all abundances given in this paper are mass fractions. Dreizler (1999) used HST/GHRS spectra to assess metal abundances in WD 0111+002. With the exception of carbon lines, only a few weak Niv lines were detected and an upper limit of $\mathrm{Ni}=1.4 \times 10^{-5}$ was established. Archival FUSE spectra of both stars remained hitherto not analyzed.

\section{2. $P G 1707+427$ (PG1159 star)}

This star is a well-studied object (Werner et al. 2015, and references therein). It has $T_{\text {eff }}=85000 \pm 5000 \mathrm{~K}$ and $\log g=$ $7.5 \pm 0.5$, and is dominated by helium and carbon in roughly equal amounts. In the work cited, we assessed the abundances of metals up to the iron group using far-UV spectra taken with the Far Ultraviolet Spectroscopic Explorer (FUSE) and HST spectra of relatively low resolution taken with the Goddard High Resolution Spectrograph (GHRS). Our new HST/COS spectroscopy aimed at spectra of better quality to detect weaker lines, particularly of heavy metals.

\section{Observations}

In Table 1, we list the observation log of our new HST/COS data together with other archival UV HST and FUSE spectra used in our study. The datasets were retrieved from the Barbara A. Mikulski Archive for Space Telescopes (MAST). According to the COS Instrument Science Report 2009-01(v1) ${ }^{1}$, the line spread function (LSF) of the COS spectra deviates from a Gaussian shape. We therefore convolve all synthetic spectra with the correct LSF.

We used optical observations to constrain the surface gravity from the ionized helium lines. Optical spectra for WD $0111+002$ and PG 1707+427 were obtained from the Sloan Digital Sky Survey (SDSS; observation IDs 1237663784742354959 and

\footnotetext{
1 http://www.stsci.edu/hst/cos/documents/isrs/ISR2009_ Q1.pdf
}

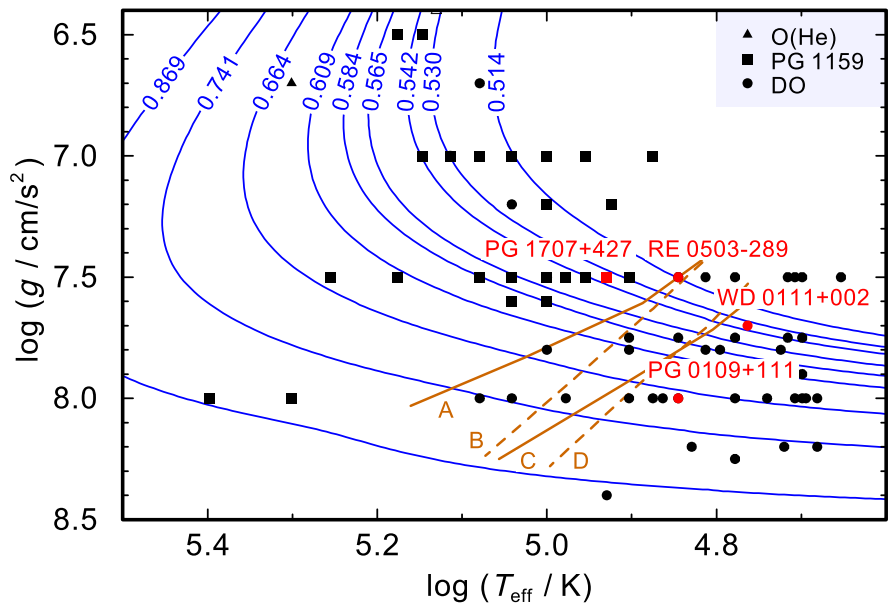

Fig. 1. Location of our three program stars and the comparison star RE 0503-289 (red symbols) together with related objects (Hügelmeyer et al. 2006; Kepler et al. 2016; Reindl et al. 2014a; Werner \& Herwig $2006)$ in the $T_{\text {eff }}-g$ plane. The single triangle symbol denotes a heliumdominated object of the $\mathrm{O}(\mathrm{He})$ spectral class (Reindl et al. 2014b). Evolutionary tracks for H-deficient WDs (Althaus et al. 2009) labeled with the respective masses in $M_{\odot}$ are plotted for comparison. Four variants of the PG 1159-DO transition limit (labeled A-D, Unglaub \& Bues 2000) are indicated.

1237665571981623391 , respectively). We also used the spectra of PG 0109+111 from Dreizler \& Werner (1996) and PG 1707+427 from Werner et al. (1991).

\subsection{Radial velocities}

To shift the observations to rest wavelength, we determined the stellar radial velocities $v_{\text {rad }}$ from the HST/COS spectra. To measure the observed wavelengths by Gaussian fits, we employed $\mathrm{IRAF}^{2}$. The average values are $v_{\mathrm{rad}}=44.8 \pm 5.4 \mathrm{~km} / \mathrm{s}, v_{\mathrm{rad}}=$ $37.2 \pm 4.7 \mathrm{~km} / \mathrm{s}$, and $v_{\mathrm{rad}}=-33.8 \pm 5.9 \mathrm{~km} / \mathrm{s}$ for $\mathrm{WD} 0111+002$ (from 11 lines), PG0109+111 (11 lines), and PG 1707+427 (32 lines), respectively. Considering error limits, our value for WD $0111+002$ is marginally in agreement with wavelength measurements of several lines in HST/GHRS spectra performed by Dreizler (1999).

2 IRAF is distributed by the National Optical Astronomy Observatory, which is operated by the Associated Universities for Research in Astronomy, Inc., under cooperative agreement with the National Science Foundation. 


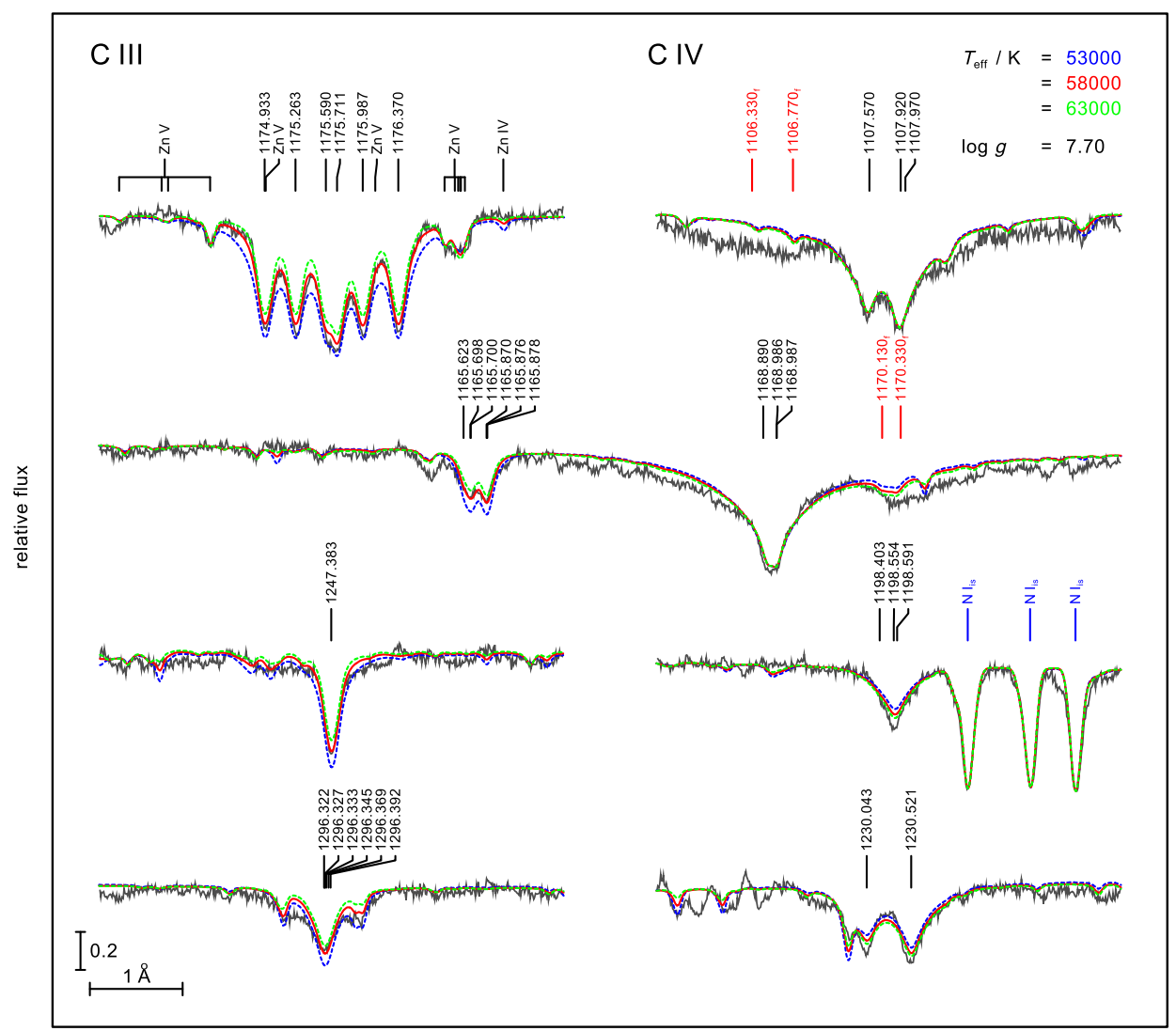

$\Delta \lambda / \AA$
Fig. 2. C III and C IV lines in the HST/COS spectrum of WD $0111+002$ (black) compared with three $\log g=7.7$ models with different $T_{\text {eff }}: 53000 \mathrm{~K}$ (blue dashed), $58000 \mathrm{~K}$ (red), and $63000 \mathrm{~K}$ (green dashed). Red wavelength labels indicate forbidden C IV line components (Werner et al. 2016). Also indicated are $\mathrm{Zn}$ lines near the $\mathrm{C}$ III multiplet at $1175 \AA$. The vertical bar indicates $20 \%$ of the continuum flux.

\subsection{Interstellar neutral hydrogen and reddening}

We used the Ly $\beta$ line profile in the FUSE spectra to measure the interstellar neutral hydrogen column density $N_{H}$ (Fig. A.1). The results are $1.1_{-0.6}^{+0.8} \times 10^{20} \mathrm{~cm}^{-2}$ for WD $0111+002$, $1.8_{-0.8}^{+0.8} \times 10^{20} \mathrm{~cm}^{-2}$ for PG $0109+111$, and $1.2_{-0.6}^{+0.8} \times 10^{20} \mathrm{~cm}^{-2}$ for PG $1707+427$. Dreizler \& Heber (1998) used the Ly $\alpha$ line in the HST/GHRS spectrum of PG 1707+427 and found $1.5 \times$ $10^{20} \mathrm{~cm}^{-2}$, that is consistent with our value within its error limit.

We converted $N_{H}$ into reddening $E_{\mathrm{B}-\mathrm{V}}$ using the empirical relationship derived by Groenewegen \& Lamers (1989): $\log \left(N_{H} / E_{\mathrm{B}-\mathrm{V}}\right)=21.58 \pm 0.10$. We obtained $E_{\mathrm{B}-\mathrm{V}}=0.029_{-0.018}^{+0.034}$ for WD $0111+002,0.047_{-0.026}^{+0.039}$ for PG 0109+111, and $0.032_{-0.019}^{+0.035}$ for PG $1707+427$. In Fig. A.2, we show our final models attenuated with these reddening values together with the observed continuum shape. The agreement is very good. To further improve the fits, marginally lower reddening values (within error limits) for WD 0111+002 and PG 1707+427 are required. Our result for PG $1707+427$ agrees with the value of 0.02 derived by Dreizler \& Heber (1998) from the continuum slope of the GHRS spectrum.

\subsection{Unidentified lines}

There are many unidentified photospheric lines in the HST/COS spectra of our program stars. Particularly conspicuous are features that are seen in both our spectrum of PG 0109+111 and in a spectrum of the comparison star RE 0503-289, taken with the Space Telescope Imaging Spectrograph (STIS) aboard HST and presented by Hoyer et al. (2017); see Fig. A.5. These features potentially stem from trans-iron elements and we therefore list their wavelength positions in Table A.1.
Table 2. Number of NLTE levels and lines in our model ions.

\begin{tabular}{|c|c|c|c|c|c|c|c|}
\hline & I & II & III & IV & V & VI & VII \\
\hline $\mathrm{He}$ & 19.35 & 16.120 & & & & & \\
\hline $\mathrm{C}$ & & & 51.217 & 54.295 & & & \\
\hline $\mathrm{N}$ & & & 34.129 & 90.546 & 54.297 & & \\
\hline $\mathrm{O}$ & & & 72.322 & 83.637 & $50.235^{a}$ & & \\
\hline $\mathrm{F}$ & & & & 30.70 & 30.104 & $30.93^{b}$ & \\
\hline $\mathrm{Ne}$ & & & 46.126 & 40.126 & $94.641^{c}$ & & \\
\hline $\mathrm{Si}$ & & & 17.28 & 30.102 & 25.59 & & \\
\hline $\mathrm{P}$ & & & & 36.28 & 18.49 & & \\
\hline $\mathrm{S}$ & & & & 57.332 & 39.107 & 25.48 & \\
\hline As & & & & 14.7 & 5.10 & 14.5 & 7.1 \\
\hline $\mathrm{Sn}$ & & & 21.9 & 10.16 & 9.5 & 15.5 & \\
\hline
\end{tabular}

Notes. First and second number of each table entry, separated by commas, denote the number of levels and lines, respectively. Not listed for each element is the highest considered ionization stage, which comprises its ground state only. See text for the treatment of iron-group and trans-iron elements besides As and Sn. ${ }^{(a)}$ For WD 0111+002, O v was the highest ionization stage with one level. ${ }^{(b)}$ Only considered for PG $1707+427 .{ }^{(c)}$ For WD $0111+002$, Ne V was the highest ionization stage with one level.

\section{Spectral analysis}

\subsection{Model atmospheres and atomic data}

To calculate chemically homogeneous, NLTE model atmospheres, we used the Tübingen model-atmosphere package (TMAP $^{3}$; Werner et al. 2003, 2012a). It assumes plane-parallel

3 http://astro.uni-tuebingen.de/ TMAP 


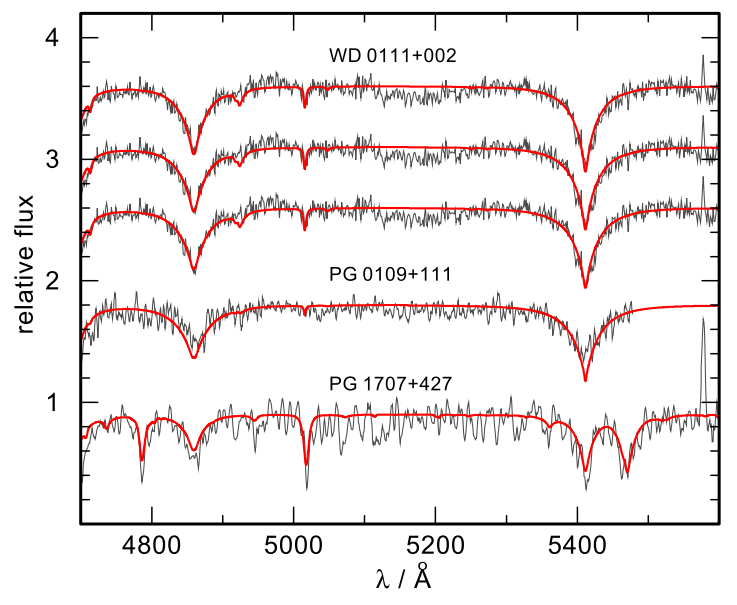

Fig. 3. Observed spectra (black) around two lines of the He II Pickering series compared with synthetic spectra (red). From top to bottom: WD $0111+002$ with three models $\left(T_{\text {eff }}=58000 \mathrm{~K}\right.$ and $\log g=7.6,7.7$, 7.8), PG 0109+111 with model $70000 / 7.5$, PG $1707+427$ with model $85000 / 7.5$. The observations of PG 0109+111 and PG 1707+427 were smoothed with a low-pass filter (Savitzky \& Golay 1964).

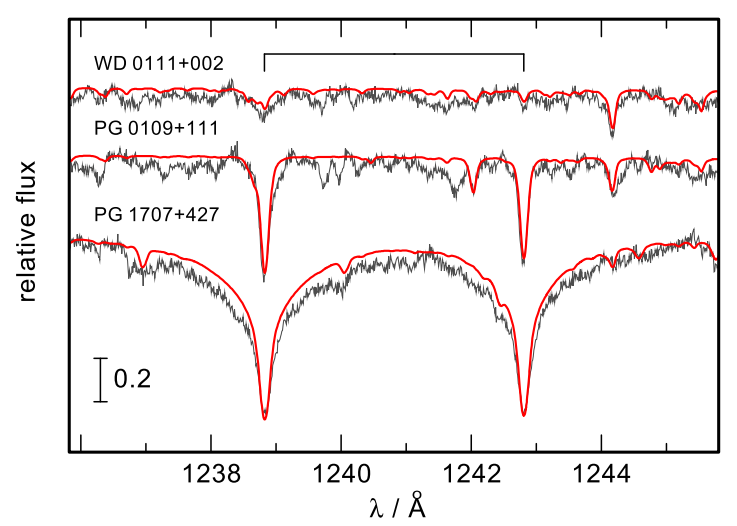

Fig. 4. N V resonance doublet in the program stars (black) compared to our final models (red).

geometry and radiative and hydrostatic equilibrium. Atomic data were compiled from the Tübingen Model-Atom Database (TMAD; Rauch \& Deetjen 2003) that has been constructed as a part of the Tübingen contribution to the German Astrophysical Virtual Observatory $\left(\mathrm{GAVO}^{4}\right)$. A summary of the model atoms for the included light elements up to atomic number $Z=16$, As and $\mathrm{Sn}$ are given in Table 2.

For iron and nickel, we used a statistical approach (employing the IrOnIc tool; Rauch \& Deetjen 2003) with seven superlevels per ion linked by superlines, together with an opacity sampling method. Ionization stages Fe III-VI (plus Fe VII for the PG 1707+427 models) and Ni III-VI augmented by a single, ground-level stage were considered per species using the line lists of Kurucz (Kurucz 1991, 2009, 2011).

Motivated by the discovery of trans-iron elements in the DO RE 0503-289 (Werner et al. 2012b), respective transition probabilities have been calculated (for references see Table A.2) and are provided by the Tübingen Oscillator Strength Service (TOSS), which is a part of the GAVO project. For our analysis, these species were treated with the same statistical method as $\mathrm{Fe}$

4 http://www.g-vo.org

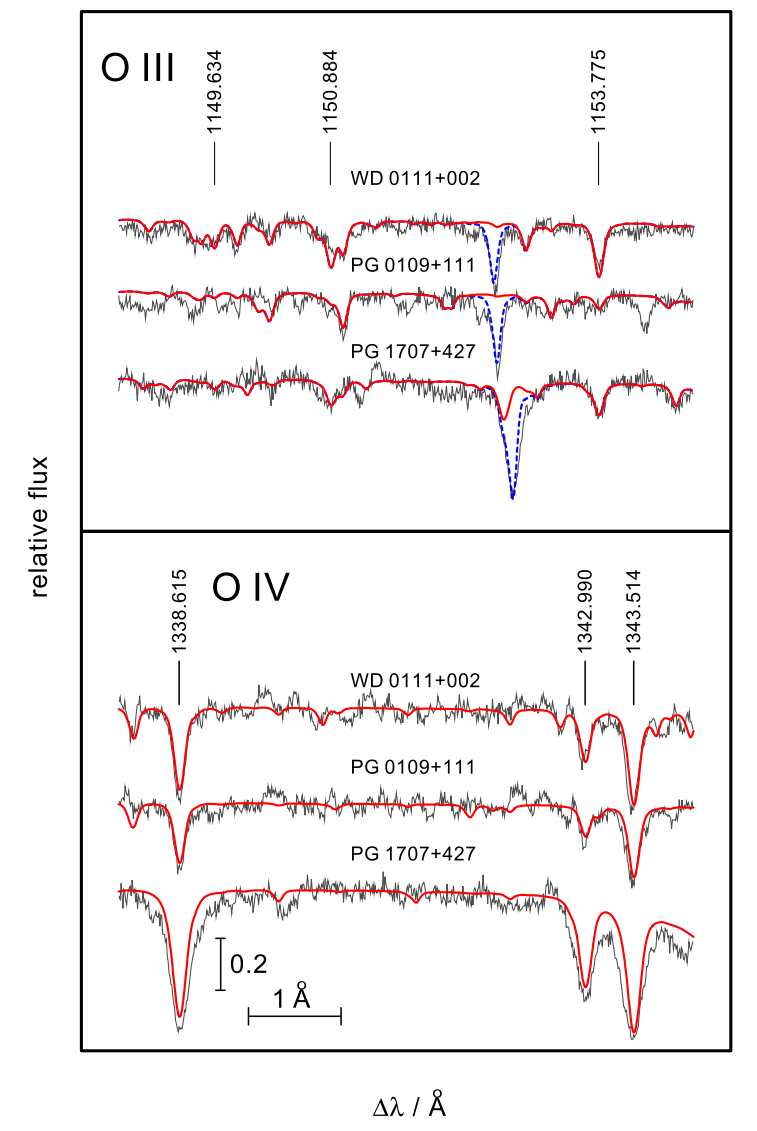

Fig. 5. Like Fig. 4, but for O III and O IV lines.

and Ni (Rauch et al. 2015b, except for As and Sn, for which classical model atoms as for the other species were used), considering lines of Zn IV-V, Ga IV-VI, Ge IV-VI, As IV-VII, Se IV-VI, Kr III-VII, Sr III-VII, Zr III-VII, Mo III-VII, Sn III-VI, Te IV-VI, I IV-VI, Xe III-VII, and Ba V-VII.

The model atmospheres for all three program stars include $\mathrm{He}, \mathrm{C}, \mathrm{N}, \mathrm{O}$, and $\mathrm{Ne}$. The neon abundance cannot be determined, so we assumed the following values: $\mathrm{Ne}=0.01$ for $\mathrm{PG} 1707+427$, which is a value that is typical for PG 1159 stars (Werner et al. 2004), and rather small values for WD 0111+002 $\left(2.0 \times 10^{-5}\right)$ and PG $0109+111\left(1.5 \times 10^{-6}\right)$. For the two hottest program stars (PG 0109+111 and PG 1707+427), we also included the following species into the model atmosphere calculations: F, Si, P, $\mathrm{S}, \mathrm{Zn}, \mathrm{Ga}$, and $\mathrm{Ge}$. To save computing time, smaller versions of the model atoms for all metals up to sulfur were used for the model atmosphere calculations. Subsequently, the large versions (whose numbers of levels and lines are listed in Table 2) were used to compute improved level occupation numbers while keeping fixed the model-atmosphere structure. All other species heavier than $\mathrm{Ge}$ were considered as trace elements, i.e., their backreaction on the model structure was neglected.

\subsection{Effective temperature and surface gravity}

To measure $T_{\text {eff }}$, we evaluated the $\mathrm{C}_{\text {III }} / \mathrm{C}_{\text {IV }}$ ionization equilibrium using the multiplets of $\mathrm{C}_{\mathrm{III}}$ at $1166,1296,1175 \AA$ plus a singlet at $1247 \AA$, and the multiplets of C IV at $1108,1169,1198$, $1230 \AA$ (Figs. 2, A.3, and A.4). For the determination of $g$ we used lines of the He II Pickering series (Fig. 3). Various other ionization equilibria ( $\mathrm{N}_{\text {III }} / \mathrm{N}_{\mathrm{IV}} / \mathrm{N}_{\mathrm{v}}, \mathrm{O}_{\mathrm{III}} / \mathrm{O}_{\mathrm{IV}} / \mathrm{O}_{\mathrm{v}} / \mathrm{O}_{\mathrm{VI}}, \mathrm{F}_{\mathrm{V}} / \mathrm{F}_{\mathrm{VI}}$, $\mathrm{S}$ v/S vi, Fe v/Fe vi/Fe vII, Zn Iv/Zn v Ge Iv/Ge v, Ga Iv/Ga v; not 


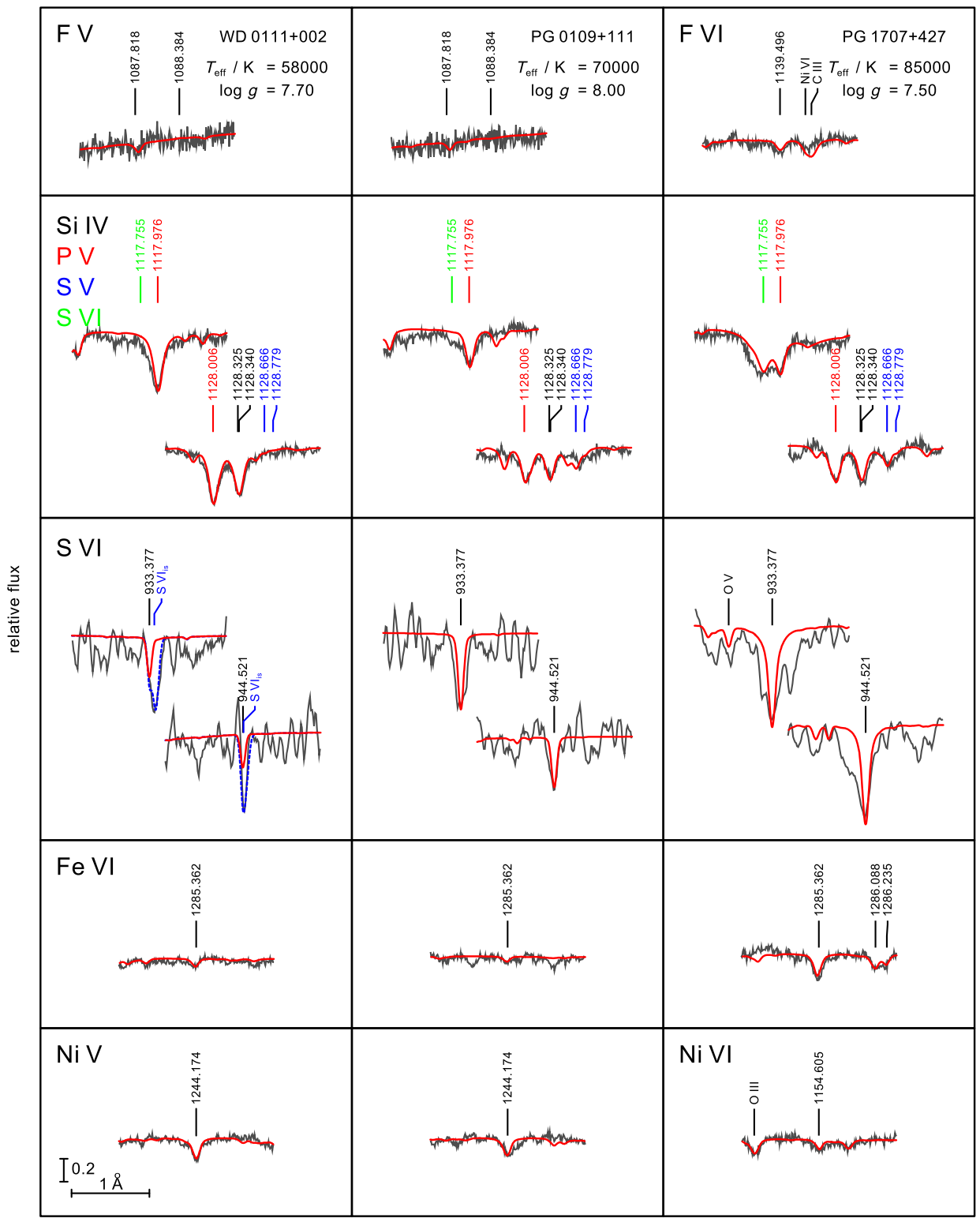

$\Delta \lambda / \AA$
Fig. 6. Synthetic line profiles for $\mathrm{F}, \mathrm{Si}, \mathrm{P}, \mathrm{S}$, $\mathrm{Fe}$, and $\mathrm{Ni}$ in our final models (red) compared to observations (black) of our three program stars. all balances available in all stars) confirm the results for $T_{\text {eff }}$ and $\log g$ found by this procedure.

For WD 0111+002, we found $T_{\text {eff }}=58000 \pm 5000 \mathrm{~K}$ and $\log g=7.7 \pm 0.3$, in agreement with previous results obtained from optical spectra alone $(65000 / 7.8$; Dreizler \& Werner 1996). In the case of PG 0109+111, we derived $T_{\text {eff }}=70000 \pm$ $5000 \mathrm{~K}$ and $\log g=8.0 \pm 0.3$. In comparison to previous results (110000/8.0; Dreizler \& Werner 1996), the temperature is significantly lower. In the work cited, it was noted that indeed the weak, observed He I lines are not compatible with the hightemperature model that displays no neutral helium lines at all. For PG $1707+427$ our results $\left(T_{\text {eff }}=85000 \pm 5000 \mathrm{~K}, \log g=\right.$ $7.5 \pm 0.3$ ) confirm previous analyses of UV and optical data (Werner et al. 2015, and references therein).

\subsection{Element abundances}

Element abundances were derived by detailed line-profile fits. Upper limits were established by reducing the abundances such that computed spectral lines become undetectable regarding the signal-to-noise ratio. We describe the procedure for the different species and display some representative fits. Results are given in Table 3 and Fig. 8. Typical errors are estimated to 0.3 dex. A comparison of the complete HST/COS spectra with the final models appears in Fig. A.5.

Carbon, nitrogen, and oxygen. For carbon, we used the same lines as for the $T_{\text {eff }}$ determination (Figs. 2, A.3, and A.4). For nitrogen, the $\mathrm{NV}$ resonance doublet is the primary indicator (Fig. 4), and for oxygen the O III $\lambda \lambda 1149.6,1150.9,1153.8 \AA$ and O IV $\lambda \lambda 1338.6,1343.0,1343.5 \AA$ lines (Fig. 5).

Fluorine, silicon, phosphorus, and sulfur. For these species, we proceeded as described in detail for the analysis of the FUSE spectrum of PG 1707+427 (Werner et al. 2015). The following lines were used: F v $\lambda \lambda 1087.8,1088.4 \AA$, F VI $\lambda 1139.5 \AA$, Si IV $\lambda \lambda 1066.6,1122.5,1128.3 \AA$, P v $\lambda \lambda 1000.4,1118.0,1128.0 \AA, \mathrm{S} v$ $\lambda \lambda 1128.7,1128.8 \AA, \mathrm{S}$ VI $\lambda \lambda 933.4,944.5,1117.8 \AA$. The fits to most of these lines are presented in Fig. 6.

Iron and nickel. Iron could not be detected in PG 0109+111, but a few weak Fe VI lines are detected in WD 0111+002. In the COS spectra of PG 1707+427, we see many lines from Fe VI and 
A\&A 612, A62 (2018)

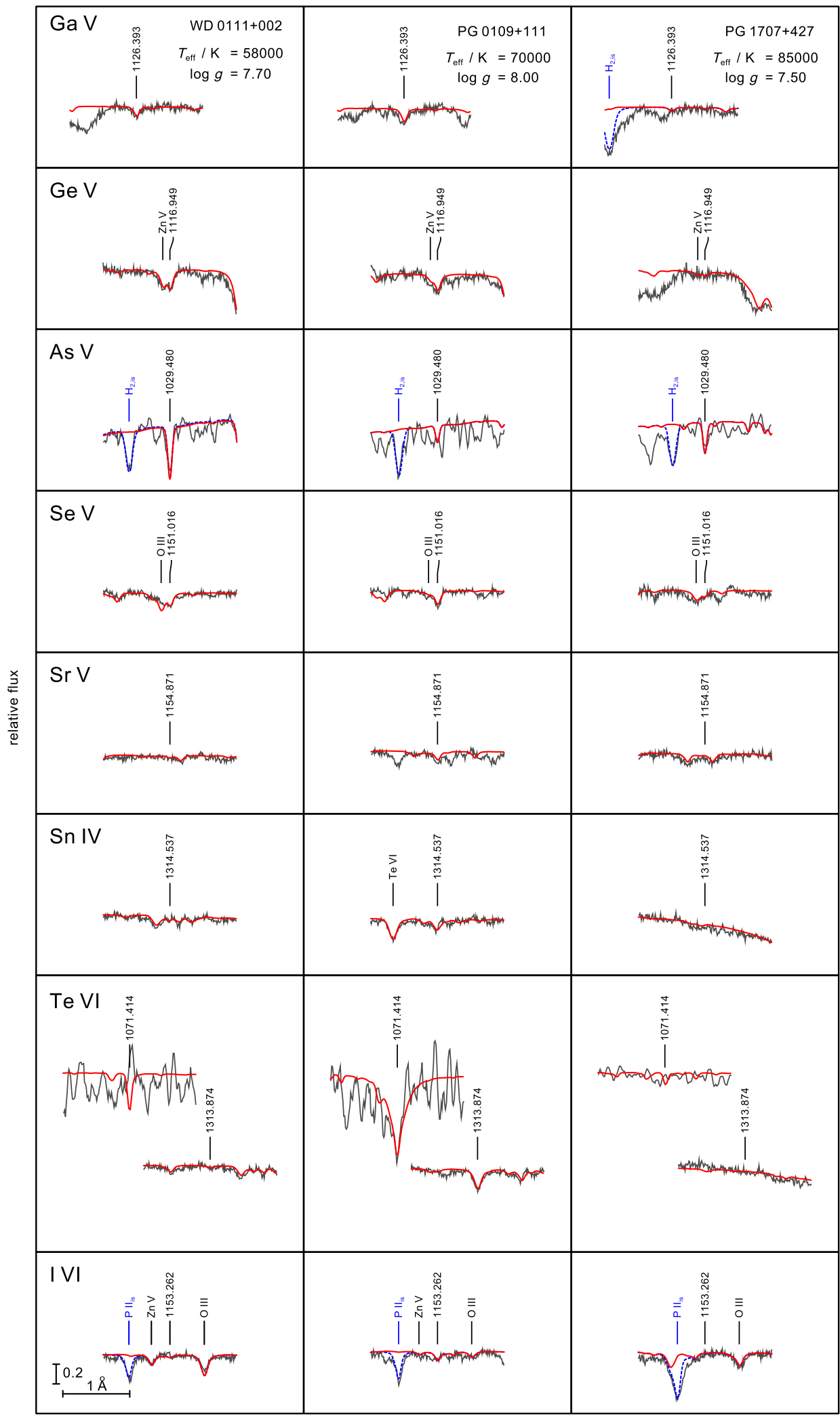

$\Delta \lambda / \AA$
Fig. 7. Like Fig. 6, but for $\mathrm{Ga}, \mathrm{Ge}, \mathrm{As}$, Se, $\mathrm{Sr}, \mathrm{Sn}, \mathrm{Te}$, and I. few from Fe VII. The identification of iron was uncertain in the FUSE spectra (Werner et al. 2015). We detect many nickel lines (Ni V) in the COS spectra of WD 0111+002 and PG 0109+111. In PG 1707+427, the presence of Ni VI lines in FUSE spectra is uncertain (Werner et al. 2015), but in the COS spectra we detect two lines at 1124.19 and $1154.60 \AA$ (Fig. 6, Tables A.3-A.5).
Zinc. Many Zn v lines are present in the COS data of our program stars; these lines are most prominent in WD 0111+002, followed by PG 0109+111, and are rather weak in PG 1707+427. Among the strongest features is a $\mathrm{Zn} \mathrm{V}$ blend at $1177 \AA$ adjacent to the $\mathrm{C}_{\text {III }} \lambda 1175 \AA$ multiplet (Figs. 2, A.3, and A.4). WD 0111+002 also shows Zn IV lines, for example, at 1349.88 $\AA$. 


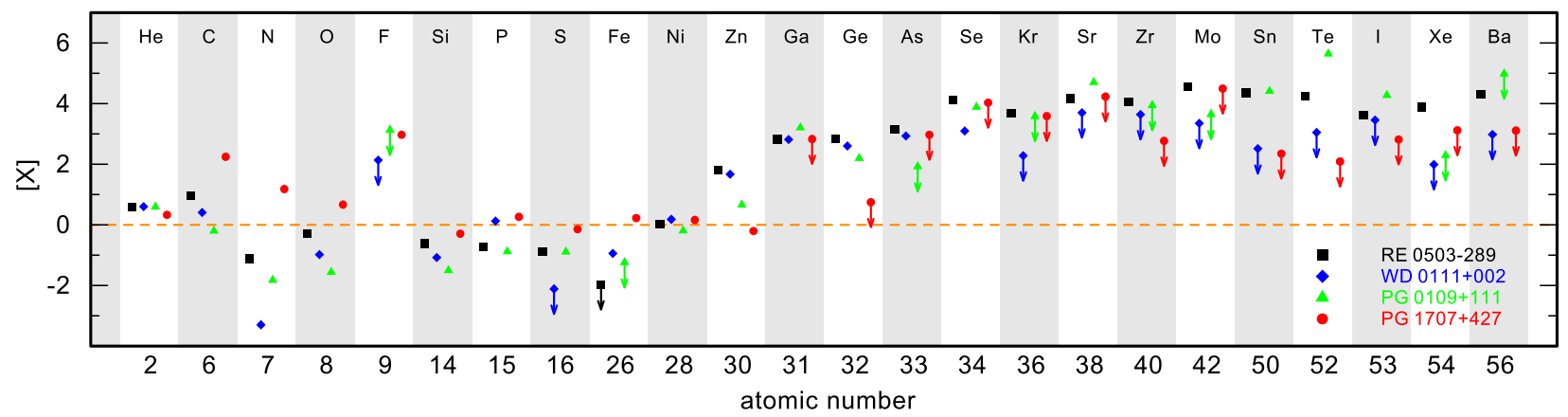

Fig. 8. Photospheric abundances of our three program stars and the comparison star RE 0503-289. [X] denotes log(abundance/solar abundance) of species X. The dashed horizontal line indicates solar abundances.

Gallium and germanium. No lines are detectable in PG 1707+427, but we see a number of Ga IV and Ga V as well as Ge IV and Ge V lines in WD 0111+002 and PG 0109+111 (Fig. 7).

As, Se, Sr Sn, Te, and I. We used the As V $\lambda \lambda 987.7 / 1029.48 \AA$ resonance doublet in the FUSE spectra to determine the As abundance in WD 0111+002 and upper limits for the other two stars. In the case of PG 1707+427, ISM lines are located at the wavelength position of the As V doublet (Fig. A.1 in Werner et al. 2015), such that we can derive an upper limit only. Se V $\lambda \lambda 1151.0$, $1227.5 \AA$ can be seen in both DOs but not in PG $1707+427$. Lines from Sr, Sn, Te, and I are only seen in PG 0109+111 (Sr V $\lambda 1154.9 \AA$, Sn IV $\lambda 1314.5 \AA$, Te VI $\lambda 1313.9 \AA$, I VI $\lambda 1153.3 \AA$ ) but not in the other two stars. For line fits, see Fig. 7.

In PG 0109+111, the fitted Sn IV $\lambda 1314.5 \AA$ line is rather weak. A number of detected Sn V lines would be better suited for a more precise analysis, but oscillator strengths are unavailable. Sn V lines are not detected in PG $1707+427$, but they would give a much tighter upper limit for the $S n$ abundance than that derived from the absence of the Sn IV line.

The tellurium abundance in PG $0109+111$ is extremely high (5.6 dex oversolar). Its mass fraction of $6.2 \times 10^{-3}$ means that it is the most abundant metal in the atmosphere. The abundance was derived from the Te VI $\lambda 1313.9 \AA$ line (see Fig. 7). In fact, there are two much stronger Te VI lines, namely the resonance doublet at 951.0 and $1071.4 \AA$, but they are located in rather noisy portions of the FUSE and HST/COS spectra. Nevertheless, these lines can be detected in the observations (see the 1071.4 $\AA$ component in Fig. A.5) as very strong and broad features. They are among the strongest metal line features rivaling the prominent $\mathrm{C}$ III and C IV lines.

$\mathrm{Kr}, \mathrm{Zr}, \mathrm{Mo}, \mathrm{Xe}$, and $\mathrm{Ba}$. No lines of these species could be identified in our program stars.

\section{Summary and conclusions}

We have analyzed new UV spectra taken with HST/COS of one PG 1159 star and two hot DO WDs. The primary aim of this study was the identification and abundance determination of trans-iron group elements to reach a conclusion about the effects of s-process and element diffusion. To this end, we computed line-blanketed NLTE model atmospheres and began with an assessment of the effective temperatures and surface gravities. For both DOs, the evaluation of metal ionization balances resulted in improved $T_{\text {eff }}$ determinations yielding lower values than found in previous analyses of optical spectra. In particular, $T_{\text {eff }}$ of PG $0109+111$ is $70000 \mathrm{~K}$ instead of $110000 \mathrm{~K}$.

PG 0109+111 and WD 0111+002 (DO WDs). For the two DOs, we measured the trace-element abundances and compare these to the DO RE 0503-289, a hitherto unique DO in which a total of 14 trans-iron group elements (i.e., atomic number $Z>28$ ) have been identified, with extreme overabundances (about 2-4.5 dex) relative to the solar values (Table 3 and Fig. 8). Both investigated DOs also exhibit trans-iron species, but there are fewer of these species (five and eight in WD 0111+002 and PG 0109+111, respectively). In both DOs we detect $\mathrm{Zn}, \mathrm{Ga}, \mathrm{Ge}$, and Se, plus the heavier species Sr, Sn, Te, and I only in PG0109+111, and As only in WD 0111+002. Generally, their abundances are (within about 1 dex) similar to RE 0503-289 with the exception of Te. This element is almost 6 dex oversolar, significantly exceeding the enrichment in RE 0503-289.

The general tendency that RE 0503-289 $\left(T_{\text {eff }}=70000 \mathrm{~K}\right.$, $\log g=7.5$ ) has the highest number of detected trans-iron species can be explained by the fact that radiative acceleration is probably more effective compared to PG0109+111, which has a higher gravity (70000/8.0), and compared to WD 0111+002 (with the lowest number of detected trans-iron species), which is significantly cooler and has a slightly higher surface gravity (58 000/7.7).

Chayer et al. (2005) detected trans-iron elements in two cool DO WDs, namely HZ21 $\left(T_{\text {eff }}=53000 \mathrm{~K}, \log g=7.8\right.$; Dreizler $\&$ Werner 1996) and HD 149499B $\left(T_{\text {eff }}=49500 \mathrm{~K}, \log g=\right.$ 7.97; Napiwotzki et al. 1995). Their abundances are also given in Table 3. The numbers listed (mass fractions) were computed from the number ratios reported in Chayer et al. (2005) (averaged over results from several lines, if applicable), accounting for the fact that HD 149499B is a DOA WD with 6\% hydrogen (by mass). Generally, the trans-iron element abundances are lower than in the hot DOs, probably owing to weaker levitation at lower temperatures.

As to the lighter elements, these are also generally less abundant in the two DOs investigated here compared to RE 0503-289, with the exception of P, that is 0.8 dex higher in WD 0111+002 than in RE 0503-289. A noteworthy commonality of all three DOs is the low $\mathrm{Fe} / \mathrm{Ni}$ ratio of $\leq 1.6$, in contrast to the solar ratio $\mathrm{Fe} / \mathrm{Ni}=18$.

PG 1707+427 (PG1159 star). Our results for $T_{\text {eff }}$ and $\log g$ agree with those in Werner et al. (2015). The same holds, within error limits, for the element abundances. For the first time, however, we identify nickel lines and find a solar $\mathrm{Ni}$ abundance. The most significant result from our new HST/COS spectroscopy 
Table 3. Final adopted atmospheric parameters of the three program stars and the comparison star RE 0503-289 (Cols. 2-5), trans-iron abundances from literature for two cool DOs (HD 149499B and HZ 21) (Cols. 6 and 7); and solar abundances (last column).

\begin{tabular}{|c|c|c|c|c|c|c|c|}
\hline & $\begin{array}{r}\text { WD } 0111 \\
+002\end{array}$ & $\begin{array}{r}\text { PG } 0109 \\
+111\end{array}$ & $\begin{array}{r}\text { PG } 1707 \\
+427\end{array}$ & $\begin{array}{r}\text { RE } 0503 \\
-289\end{array}$ & $\begin{array}{r}\text { HD } \\
149499 B\end{array}$ & HZ 21 & Sun \\
\hline Type & DO & DO & PG1159 & DO & DOA & DO & \\
\hline$T_{\mathrm{eff}} / \mathrm{K}$ & 58000 & 70000 & 85000 & 70000 & 49500 & 53000 & \\
\hline $\log g$ & 7.7 & 8.0 & 7.5 & 7.5 & 7.97 & 7.8 & \\
\hline $\mathrm{He}$ & $9.9 \times 10^{-1}$ & $9.9 \times 10^{-1}$ & $5.3 \times 10^{-1}$ & $9.7 \times 10^{-1}$ & & & $2.5 \times 10^{-1}$ \\
\hline $\mathrm{C}$ & $6.0 \times 10^{-3}$ & $1.5 \times 10^{-3}$ & $4.1 \times 10^{-1}$ & $2.2 \times 10^{-2}$ & & & $2.4 \times 10^{-3}$ \\
\hline $\mathrm{N}$ & $3.5 \times 10^{-7}$ & $1.0 \times 10^{-5}$ & $1.0 \times 10^{-2}$ & $5.5 \times 10^{-5}$ & & & $6.9 \times 10^{-4}$ \\
\hline $\mathrm{O}$ & $6.0 \times 10^{-4}$ & $1.6 \times 10^{-4}$ & $2.7 \times 10^{-2}$ & $2.9 \times 10^{-3}$ & & & $5.7 \times 10^{-3}$ \\
\hline $\mathrm{F}$ & $<4.7 \times 10^{-5}$ & $<4.7 \times 10^{-4}$ & $3.2 \times 10^{-4}$ & & & & $3.5 \times 10^{-7}$ \\
\hline $\mathrm{Si}$ & $5.6 \times 10^{-5}$ & $2.1 \times 10^{-5}$ & $3.4 \times 10^{-4}$ & $1.6 \times 10^{-4}$ & & & $6.7 \times 10^{-4}$ \\
\hline $\mathrm{P}$ & $7.7 \times 10^{-6}$ & $7.6 \times 10^{-7}$ & $1.1 \times 10^{-5}$ & $1.1 \times 10^{-6}$ & & & $5.8 \times 10^{-6}$ \\
\hline $\mathrm{S}$ & $<2.4 \times 10^{-6}$ & $4.0 \times 10^{-5}$ & $2.2 \times 10^{-4}$ & $4.0 \times 10^{-5}$ & & & $3.1 \times 10^{-4}$ \\
\hline $\mathrm{Fe}$ & $1.4 \times 10^{-4}$ & $<6.9 \times 10^{-5}$ & $2.0 \times 10^{-3}$ & $<1.3 \times 10^{-5}$ & & & $1.2 \times 10^{-3}$ \\
\hline $\mathrm{Ni}$ & $1.0 \times 10^{-4}$ & $4.3 \times 10^{-5}$ & $9.9 \times 10^{-5}$ & $7.2 \times 10^{-5}$ & & & $6.8 \times 10^{-5}$ \\
\hline $\mathrm{Zn}$ & $8.2 \times 10^{-5}$ & $8.1 \times 10^{-6}$ & $1.1 \times 10^{-6}$ & $1.1 \times 10^{-4}$ & & & $1.7 \times 10^{-6}$ \\
\hline $\mathrm{Ga}$ & $3.5 \times 10^{-5}$ & $8.6 \times 10^{-5}$ & $<3.6 \times 10^{-5}$ & $3.4 \times 10^{-5}$ & & & $5.3 \times 10^{-8}$ \\
\hline $\mathrm{Ge}$ & $9.1 \times 10^{-5}$ & $3.6 \times 10^{-5}$ & $<1.3 \times 10^{-6}$ & $1.6 \times 10^{-4}$ & & & $2.3 \times 10^{-7}$ \\
\hline As & $9.3 \times 10^{-6}$ & $<9.2 \times 10^{-7}$ & $<1.0 \times 10^{-5}$ & $1.6 \times 10^{-5}$ & $8.1 \times 10^{-6}$ & $3.0 \times 10^{-7}$ & $1.1 \times 10^{-8}$ \\
\hline $\mathrm{Se}$ & $1.6 \times 10^{-4}$ & $9.6 \times 10^{-4}$ & $<1.3 \times 10^{-3}$ & $1.6 \times 10^{-3}$ & $1.3 \times 10^{-5}$ & $<7.5 \times 10^{-6}$ & $1.3 \times 10^{-7}$ \\
\hline $\mathrm{Kr}$ & $<2.1 \times 10^{-5}$ & $<4.1 \times 10^{-4}$ & $<4.2 \times 10^{-4}$ & $5.0 \times 10^{-4}$ & & & $1.1 \times 10^{-7}$ \\
\hline $\mathrm{Sr}$ & $<2.2 \times 10^{-4}$ & $2.2 \times 10^{-3}$ & $<7.3 \times 10^{-4}$ & $6.5 \times 10^{-4}$ & & & $4.3 \times 10^{-8}$ \\
\hline $\mathrm{Zr}$ & $<1.1 \times 10^{-4}$ & $<2.3 \times 10^{-4}$ & $<1.5 \times 10^{-5}$ & $3.0 \times 10^{-4}$ & & & $2.6 \times 10^{-8}$ \\
\hline Mo & $<1.2 \times 10^{-5}$ & $<2.4 \times 10^{-5}$ & $<1.7 \times 10^{-4}$ & $1.9 \times 10^{-4}$ & & & $5.3 \times 10^{-9}$ \\
\hline $\mathrm{Sn}$ & $<2.9 \times 10^{-6}$ & $2.3 \times 10^{-4}$ & $<2.0 \times 10^{-6}$ & $2.0 \times 10^{-4}$ & $8.3 \times 10^{-6}$ & & $9.1 \times 10^{-9}$ \\
\hline $\mathrm{Te}$ & $<1.6 \times 10^{-5}$ & $6.2 \times 10^{-3}$ & $<1.7 \times 10^{-6}$ & $2.5 \times 10^{-4}$ & $9.1 \times 10^{-7}$ & $1.3 \times 10^{-6}$ & $1.4 \times 10^{-8}$ \\
\hline I & $<9.4 \times 10^{-6}$ & $6.2 \times 10^{-5}$ & $<2.2 \times 10^{-6}$ & $1.4 \times 10^{-5}$ & $1.9 \times 10^{-5}$ & $<1.2 \times 10^{-6}$ & $3.3 \times 10^{-9}$ \\
\hline $\mathrm{Xe}$ & $<1.6 \times 10^{-6}$ & $<3.2 \times 10^{-6}$ & $<2.2 \times 10^{-5}$ & $1.3 \times 10^{-4}$ & & & $1.7 \times 10^{-8}$ \\
\hline $\mathrm{Ba}$ & $<1.7 \times 10^{-5}$ & $<1.7 \times 10^{-3}$ & $<2.3 \times 10^{-5}$ & $3.6 \times 10^{-4}$ & & & $1.8 \times 10^{-8}$ \\
\hline
\end{tabular}

Notes. Abundances in mass fractions (see also Fig. 8) and surface gravity $g$ in $\mathrm{cm} \mathrm{s}^{-2}$. Parameters for RE 0503-289 from Rauch et al. (2017b) and references therein. Trans-iron abundances for HD 149499B and HZ 21 from Chayer et al. (2005). Solar abundances from Asplund et al. (2009); Scott et al. (2015a,b); and Grevesse et al. (2015).

concerns the trans-iron elements. While only high upper abundance limits could be derived previously, we now determined the $\mathrm{Zn}$ abundance and, for most other species, were able to reduce the upper abundance limits significantly compared to our previous work involving FUSE data alone. We find that $\mathrm{Zn}$ is solar and $\mathrm{Ge}$ is at most 0.8 dex oversolar, suggesting that there is no general overabundance of trans-iron elements. The upper limits for other trans-iron elements are still high, however, between about 2.8 and 4.5 dex oversolar. In comparison to the DOs, this confirms that radiative levitation does not affect the element abundances in the atmosphere of PG 1707+427. This is a plausible result for a PG 1159 star because, as outlined in the introduction, the star is located before the wind limit so that diffusion cannot affect the element abundances. In addition, from the helium and light metal abundances we concluded to see intershell matter composition (Werner et al. 2015).

It is expected from stellar evolution models that, as discussed in detail in Werner et al. (2015) based on the models of a $2 M_{\odot}$ star after the 30th thermal pulse (Gallino, priv. comm.; Karakas et al. 2007) s-process elements should be overabundant. But our results do not allow a quantitative comparison because the predicted enhancement of $\mathrm{Zn}$ and $\mathrm{Ge}$ to $<0.4 \mathrm{dex}$ oversolar values is near the error margin of our analysis. Much stronger enhancements are predicted by stellar models for other trans-iron elements, but they are still below our upper limits derived. For example, we could reduce the upper detection limit for germanium by 1.6 dex down to $\mathrm{Ge}<1.3 \times 10^{-6}$, but the predicted value is $\mathrm{Ge}<5.9 \times 10^{-7}$, i.e., just barely below our detection threshold. The strongest enhancement among the investigated s-process elements is predicted for barium, namely $\mathrm{Ba}=1.2 \times 10^{-5}$. Again, although we were able to reduce the detection threshold by 1.8 dex, the current limit of $\mathrm{Ba}<2.3 \times$ $10^{-5}$ is slightly smaller. Further progress can only be achieved with UV spectra with even better signal-to-noise ratio.

Acknowledgements. DH was supported by the German Aerospace Center (DLR, grant 50OR 1501). The authors acknowledge support by the High Performance and Cloud Computing Group at the Zentrum für Datenverarbeitung of the University of Tübingen, the state of Baden-Württemberg through bwHPC, and the German Research Foundation (DFG) through grant no INST 37/935-1 FUGG. The TIRO (http://astro-uni-tuebingen.de/ TIR0) TMAD (http://astro-uni-tuebingen.de/ TMAD) and TOSS (http:// astro-uni-tuebingen.de/ TOSS) services used for this paper were constructed as part of the activities of the German Astrophysical Virtual Observatory. Some of the data presented in this paper were obtained from the Mikulski Archive for Space Telescopes (MAST). STScI is operated by the Association 
of Universities for Research in Astronomy, Inc., under NASA contract NAS526555. Support for MAST for non-HST data is provided by the NASA Office of Space Science via grant NNX09AF08G and by other grants and contracts. This research has made use of NASA's Astrophysics Data System and the SIMBAD database, operated at CDS, Strasbourg, France.

\section{References}

Althaus, L. G., Panei, J. A., Miller Bertolami, M. M., et al. 2009, ApJ, 704, 1605 Asplund, M., Grevesse, N., Sauval, A. J., \& Scott, P. 2009, ARA\&A, 47, 481 Chayer, P., Vennes, S., Dupuis, J., \& Kruk, J. W. 2005, ApJ, 630, L169 Dreizler, S. 1999, A\&A, 352, 632

Dreizler, S., \& Heber, U. 1998, A\&A, 334, 618

Dreizler, S., \& Werner, K. 1996, A\&A, 314, 217

Grevesse, N., Scott, P., Asplund, M., \& Sauval, A. J. 2015, A\&A, 573, A27

Groenewegen, M. A. T., \& Lamers, H. J. G. L. M. 1989, A\&AS, 79, 359

Hoyer, D., Rauch, T., Werner, K., Kruk, J. W., \& Quinet, P. 2017, A\&A, 598, A 135

Hügelmeyer, S. D., Dreizler, S., Homeier, D., et al. 2006, A\&A, 454, 617

Karakas, A. I., Lugaro, M., \& Gallino, R. 2007, ApJ, 656, L73

Kepler, S. O., Pelisoli, I., Koester, D., et al. 2016, MNRAS, 455, 3413

Kurucz, R. L. 1991, in 341: Stellar Atmospheres - Beyond Classical Models eds L. Crivellari, I. Hubeny, \& D. G. Hummer NATO ASIC Proc., 441

Kurucz, R. L. 2009, in American Institute of Physics Conference Series, eds. I. Hubeny, J. M. Stone, K. MacGregor, \& K. Werner, 1171, 43-51

Kurucz, R. L. 2011, Can. J. Phys., 89, 417

Napiwotzki, R., Hurwitz, M., Jordan, S., et al. 1995, A\&A, 300, L5

Rauch, T., \& Deetjen, J. L. 2003, in Stellar Atmosphere Modeling, eds. I. Hubeny, D. Mihalas, \& K. Werner, ASP Conf. Ser., 288, 103
Rauch, T., Werner, K., Biémont, É., Quinet, P., \& Kruk, J. W. 2012, A\&A, 546, A55

Rauch, T., Werner, K., Quinet, P., \& Kruk, J. W. 2014a, A\&A, 564, A41

Rauch, T., Werner, K., Quinet, P., \& Kruk, J. W. 2014b, A\&A, 566, A10

Rauch, T., Hoyer, D., Quinet, P., Gallardo, M., \& Raineri, M. 2015a, A\&A, 577, A88

Rauch, T., Werner, K., Quinet, P., \& Kruk, J. W. 2015b, A\&A, 577, A6

Rauch, T., Quinet, P., Hoyer, D., et al. 2016a, A\&A, 587, A39

Rauch, T., Quinet, P., Hoyer, D., et al. 2016b, A\&A, 590, A128

Rauch, T., Gamrath, S., Quinet, P., et al. 2017a, A\&A, 599, A142

Rauch, T., Quinet, P., Knörzer, M., et al. 2017b, A\&A, 606, A105

Reindl, N., Rauch, T., Werner, K., et al. 2014a, A\&A, 572, A117

Reindl, N., Rauch, T., Werner, K., Kruk, J. W., \& Todt, H. 2014b, A\&A, 566, A116

Savitzky, A., \& Golay, M. J. E. 1964, Anal. Chem., 36, 1627

Scott, P., Asplund, M., Grevesse, N., Bergemann, M., \& Sauval, A. J. 2015a, A\&A, 573, A26

Scott, P., Grevesse, N., Asplund, M., et al. 2015b, A\&A, 573, A25

Unglaub, K., \& Bues, I. 2000, A\&A, 359, 1042

Werner, K., \& Herwig, F. 2006, PASP, 118, 183

Werner, K., Heber, U., \& Hunger, K. 1991, A\&A, 244, 437

Werner, K., Deetjen, J. L., Dreizler, S., et al. 2003, in Stellar Atmosphere Modeling, eds. I. Hubeny, D. Mihalas, \& K. Werner, ASP Conf. Ser., 288, 31

Werner, K., Rauch, T., Reiff, E., Kruk, J. W., \& Napiwotzki, R. 2004, A\&A, 427, 685

Werner, K., Dreizler, S., \& Rauch, T. 2012a, TMAP: Tübingen NLTE ModelAtmosphere Package, Astrophysics Source Code Library

Werner, K., Rauch, T., Ringat, E., \& Kruk, J. W. 2012b, ApJ, 753, L7

Werner, K., Rauch, T., \& Kruk, J. W. 2015, A\&A, 582, A94

Werner, K., Rauch, T., Hoyer, D., \& Quinet, P. 2016, ApJ, 827, L4 


\section{Appendix A: Additional figures and tables}

Table A.1. Wavelengths (in $\AA$ ) of unidentified photospheric lines.

$1102.15,1103.28,1103.47,1104.20,1114.10,1119.20,1120.85,1122.14$ $1123.52,1137.22,1157.55,1157.90,1159.79,1161.88,1165.30,1174.66$ $1178.61,1201.28,1201.53,1234.32,1239.72,1241.78,1247.71,1250.30$ $1254.09,1259.91,1262.26,1264.11,1274.72,1278.18,1278.92,1345.67$ 1346.16

Notes. These possibly stem from trans-iron elements and are present in both, the HST/COS spectrum of PG 0109+111 and the HST/STIS spectrum of RE 0503-289.

Table A.2. Ions of trans-iron elements with recently calculated oscillator strengths.

\begin{tabular}{rrll}
\hline \hline Zn & IV & - V & Rauch et al. (2014a) \\
Ga & IV & - VI & Rauch et al. (2015b) \\
$\mathrm{Ge}$ & V & - VI & Rauch et al. (2012) \\
$\mathrm{Se}$ & V & & Rauch et al. (2017b) \\
$\mathrm{Kr}$ & IV & - VII & Rauch et al. (2016b) \\
$\mathrm{Sr}$ & IV & - VII & Rauch et al. (2017b) \\
$\mathrm{Zr}$ & IV & - VII & Rauch et al. (2017a) \\
$\mathrm{Mo}$ & IV & - VII & Rauch et al. (2016a) \\
$\mathrm{Te}$ & VI & & Rauch et al. (2017b) \\
I & VI & & Rauch et al. (2017b) \\
$\mathrm{Xe}$ & IV & - V, VII & Rauch et al. (2015a, 2017a) \\
$\mathrm{Ba}$ & V & - VII & Rauch et al. (2014b) \\
\hline
\end{tabular}

Table A.3. Fe $\mathrm{V}$ lines identified in the HST/COS spectrum of PG $1707+427$.

\begin{tabular}{|c|c|c|c|c|}
\hline \multirow{2}{*}{$\begin{array}{c}\text { Wavelength / } \AA \\
\text { laboratory }\end{array}$} & \multirow[t]{2}{*}{ Ion } & \multicolumn{2}{|c|}{ Transition } & \multirow[t]{2}{*}{ Comment } \\
\hline & & low & up & \\
\hline 1320.409 & $\mathrm{Fe} \mathrm{V}$ & $4 \mathrm{~s}^{3} \mathrm{H}_{4}$ & $4 p^{3} G_{3}^{o}$ & weak \\
\hline 1321.341 & $\mathrm{Fe} v$ & $4 s^{3} G_{4}^{4}$ & $4 \mathrm{p}^{3} \mathrm{H}_{5}^{\mathrm{O}}$ & weak \\
\hline 1321.489 & $\mathrm{Fe} \mathrm{v}$ & $4 s^{3} G_{5}^{4}$ & $4 \mathrm{p}^{3} \mathrm{H}_{6}^{\mathrm{o}}$ & weak \\
\hline 1323.271 & $\mathrm{Fe} \mathrm{v}$ & $4 \mathrm{~s}^{3} \mathrm{H}_{5}$ & $4 p^{3} G_{4}^{o}$ & weak \\
\hline 1330.405 & $\mathrm{Fe} \mathrm{v}$ & $4 \mathrm{~s}^{3} \mathrm{H}_{6}$ & $4 p^{3} G_{5}^{o}$ & \\
\hline 1331.639 & $\mathrm{Fe} \mathrm{v}$ & $4 s^{1} D_{2}$ & $4 p^{1} ?_{2}^{o}$ & \\
\hline 1361.278 & $\mathrm{Fe} \mathrm{v}$ & $4 s^{3} P_{2}^{2}$ & $4 p^{3} F_{3}^{o}$ & weak \\
\hline 1361.446 & $\mathrm{Fe} \mathrm{v}$ & $4 \mathrm{~s}{ }^{1} \mathrm{~F}_{3}$ & $4 \mathrm{p}^{1} \mathrm{G}_{4}^{\mathrm{o}}$ & weak \\
\hline 1361.691 & $\mathrm{Fe} v$ & $4 s^{1} D_{2}$ & $4 p^{1} F_{3}^{o}$ & \\
\hline 1361.826 & $\mathrm{Fe} \mathrm{v}$ & $4 s^{3} F_{4}^{2}$ & $4 \mathrm{p}^{3} \mathrm{G}_{5}^{\mathrm{o}}$ & \\
\hline 1362.864 & $\mathrm{Fe} \mathrm{v}$ & $4 s^{3} D_{3}$ & $4 p^{3} D_{3}^{o}$ & weak \\
\hline 1363.076 & $\mathrm{Fe} \mathrm{v}$ & $4 d^{5} \mathrm{~F}_{3}$ & $4 p^{5} \mathrm{~F}_{4}^{\mathrm{o}}$ & weak \\
\hline
\end{tabular}

Table A.4. Fe VI lines identified in the HST/COS spectrum of PG $1707+427$.

\begin{tabular}{|c|c|c|c|c|}
\hline \multirow{2}{*}{$\begin{array}{l}\text { Wavelength / } \\
\text { Å laboratory }\end{array}$} & \multirow[t]{2}{*}{ Ion } & \multicolumn{2}{|c|}{ Transition } & \multirow[t]{2}{*}{ Comment } \\
\hline & & low & up & \\
\hline 1115.099 & $\mathrm{Fe}$ VI & $4 s^{2} D_{5 / 2}$ & $4 p^{2} D_{5 / 2}^{o}$ & \\
\hline 1120.933 & $\mathrm{Fe}$ VI & $4 s^{2} F_{7 / 2}^{3 / 2}$ & $4 p^{2} \mathrm{~F}_{7 / 2}^{\mathrm{o}}$ & \\
\hline 1121.147 & $\mathrm{Fe}$ VI & $4 s^{2} F_{5 / 2}$ & $4 p^{2} \mathrm{~F}_{5 / 2}^{\mathrm{o}}$ & \\
\hline 1152.771 & $\mathrm{Fe}$ VI & $4 s^{2} G_{7 / 2}$ & $4 p^{2} \mathrm{~F}_{5 / 2}^{\mathrm{o}}$ & blend P II i.s. \\
\hline 1160.509 & $\mathrm{Fe}$ VI & $4 \mathrm{~s}^{2} \mathrm{P}_{1 / 2}$ & $4 \mathrm{p}^{2} \mathrm{P}_{1 / 2}^{\mathrm{o}}$ & \\
\hline 1165.674 & $\mathrm{Fe}$ VI & $4 \mathrm{~s}^{2} \mathrm{P}_{3 / 2}^{1 / 2}$ & $4 \mathrm{p}^{2} \mathrm{P}_{3 / 2}^{\mathrm{o}}$ & \\
\hline 1167.695 & $\mathrm{Fe}$ VI & $4 s^{2} G_{9 / 2}$ & $4 p^{2} \mathrm{~F}_{7 / 2}^{\mathrm{o}}$ & \\
\hline 1206.041 & $\mathrm{Fe}$ VI & $4 s^{4} P_{1 / 2}$ & $4 p^{4} \mathrm{P}_{3 / 2}^{o}$ & uncertain \\
\hline 1227.883 & $\mathrm{Fe}$ VI & $4 s^{4} P_{5 / 2}^{1 / 2}$ & $4 p^{4} P_{3 / 2}^{o}$ & uncertain \\
\hline 1228.605 & $\mathrm{Fe}$ VI & $4 s^{2} G_{9 / 2}$ & $4 \mathrm{p}^{2} \mathrm{H}_{11 / 2}^{\mathrm{o}}$ & uncertain \\
\hline 1228.681 & $\mathrm{Fe}$ VI & $4 s^{2} D_{3 / 2}$ & $4 p^{2} \mathrm{D}_{5 / 2}^{\mathrm{o}}$ & uncertain \\
\hline 1228.962 & $\mathrm{Fe}$ VI & $4 s^{4} F_{3 / 2}^{3 / 2}$ & $4 p^{4} D_{3 / 2}^{o / 2}$ & \\
\hline 1228.977 & $\mathrm{Fe}$ VI & $4 \mathrm{~s}^{2} \mathrm{D}_{5 / 2}$ & $4 p^{4} D_{7 / 2}^{o}$ & \\
\hline 1232.477 & $\mathrm{Fe}$ VI & $4 s^{4} F_{7 / 2}^{5 / 2}$ & $4 p^{4} D_{5 / 2}^{o}$ & \\
\hline 1236.973 & $\mathrm{Fe}$ VI & $4 s^{4} F_{5 / 2}$ & $4 p^{4} D_{3 / 2}^{o}$ & uncertain \\
\hline 1246.835 & $\mathrm{Fe}$ VI & $4 s^{4} F_{5 / 2}$ & $4 p^{2} \mathrm{~F}_{5 / 2}^{\mathrm{o}}$ & weak \\
\hline 1252.769 & $\mathrm{Fe}$ VI & $4 \mathrm{~s}^{4} \mathrm{P}_{3 / 2}$ & $4 p^{2} D_{3 / 2}^{o}$ & \\
\hline 1252.789 & $\mathrm{Fe}$ VI & $4 s^{2} F_{5 / 2}$ & $4 p^{2} G_{7 / 2}^{o}$ & \\
\hline 1253.045 & $\mathrm{Fe}$ VI & $4 \mathrm{~s}^{2} \mathrm{D}_{5 / 2}$ & $4 p^{4} D_{5 / 2}^{o}$ & \\
\hline 1253.675 & $\mathrm{Fe}$ VI & $4 s^{2} G_{7 / 2}$ & $4 \mathrm{p}^{2} \mathrm{H}_{9 / 2}^{\mathrm{o}}$ & blend S II i.s. \\
\hline 1258.021 & $\mathrm{Fe}$ VI & $4 s^{4} F_{9 / 2}$ & $4 p^{2} F_{7 / 2}^{o}$ & \\
\hline 1258.881 & $\mathrm{Fe}$ VI & $4 \mathrm{~s}^{4} \mathrm{~F}_{7 / 2}$ & $4 p^{2} \mathrm{~F}_{5 / 2}^{o}$ & \\
\hline 1260.746 & $\mathrm{Fe}$ VI & $4 s^{4} P_{5 / 2}$ & $4 p^{4} D_{7 / 2}^{o}$ & blend C I, Si II i.s. \\
\hline 1261.058 & $\mathrm{Fe}$ VI & $4 s^{4} F_{7 / 2}$ & $4 p^{4} F_{9 / 2}^{o}$ & \\
\hline 1265.872 & $\mathrm{Fe}$ VI & $4 s^{4} F_{5 / 2}$ & $4 p^{4} \mathrm{~F}_{7 / 2}^{o}$ & \\
\hline 1266.103 & $\mathrm{Fe}$ VI & $4 s^{2} D_{5 / 2}$ & $4 \mathrm{p}^{2} \mathrm{~F}_{7 / 2}^{\mathrm{o}}$ & \\
\hline 1271.099 & $\mathrm{Fe}$ VI & $4 s^{4} P_{3 / 2}$ & $4 p^{4} D_{5 / 2}^{o}$ & \\
\hline 1272.066 & $\mathrm{Fe}$ VI & $4 s^{4} F_{9 / 2}$ & $4 p^{4} G_{11 / 2}^{o}$ & \\
\hline 1273.843 & $\mathrm{Fe}$ VI & $4 s^{4} F_{3 / 2}$ & $4 p^{4} \mathrm{~F}_{5 / 2}^{\mathrm{o}}$ & \\
\hline 1276.877 & $\mathrm{Fe}$ VI & $4 s^{4} F_{9 / 2}$ & $4 p^{4} F_{9 / 2}^{o}$ & \\
\hline 1277.069 & $\mathrm{Fe}$ VI & $4 s^{4} P_{1 / 2}$ & $4 p^{4} D_{3 / 2}^{o}$ & \\
\hline 1278.292 & $\mathrm{Fe}$ VI & $4 s{ }^{4} F_{7 / 2}$ & $4 p^{4} F_{7 / 2}^{o}$ & \\
\hline 1282.452 & $\mathrm{Fe}$ VI & $4 s^{4} F_{5 / 2}$ & $4 p^{4} \mathrm{~F}_{5 / 2}^{\mathrm{o}}$ & \\
\hline 1285.362 & $\mathrm{Fe}$ VI & $4 s^{4} F_{7 / 2}$ & $4 p^{4} G_{9 / 2}^{o}$ & \\
\hline 1286.088 & $\mathrm{Fe}$ VI & $4 s^{4} P_{5 / 2}$ & $4 p^{4} D_{5 / 2}^{o}$ & \\
\hline 1286.235 & $\mathrm{Fe}$ VI & $4 s^{4} P_{3 / 2}$ & $4 p^{4} D_{3 / 2}^{o}$ & \\
\hline 1287.028 & $\mathrm{Fe}$ VI & $4 s^{4} F_{3 / 2}$ & $4 p^{4} F_{3 / 2}^{o}$ & \\
\hline 1291.437 & $\mathrm{Fe}$ VI & $4 s^{2} D_{5 / 2}^{3 / 2}$ & $4 p^{2} \mathrm{~F}_{5 / 2}^{\mathrm{o}}$ & \\
\hline 1292.643 & $\mathrm{Fe}$ VI & $4 s^{4} P_{3 / 2}$ & $4 p^{4} D_{1 / 2}^{o}$ & \\
\hline 1294.549 & $\mathrm{Fe}$ VI & $4 s^{4} F_{9 / 2}$ & $4 p^{4} F_{7 / 2}^{o}$ & \\
\hline 1295.201 & $\mathrm{Fe}$ VI & $4 s^{4} F_{7 / 2}$ & $4 p^{4} \mathrm{~F}_{5 / 2}^{o}$ & \\
\hline 1295.817 & $\mathrm{Fe}$ VI & $4 s^{4} F_{5 / 2}$ & $4 p^{4} F_{3 / 2}^{o}$ & \\
\hline 1296.734 & $\mathrm{Fe}$ VI & $4 s^{2} D_{3 / 2}$ & $4 p^{2} \mathrm{~F}_{5 / 2}^{\mathrm{o}}$ & \\
\hline 1296.872 & $\mathrm{Fe}$ VI & $4 s^{4} F_{5 / 2}$ & $4 p^{4} G_{7 / 2}^{o}$ & \\
\hline 1299.848 & $\mathrm{Fe}$ VI & $4 \mathrm{~s}{ }^{4} \mathrm{P}_{5 / 2}$ & $4 p^{2} \mathrm{~F}_{7 / 2}^{\mathrm{o}}$ & \\
\hline 1301.174 & $\mathrm{Fe}$ VI & $4 \mathrm{~s}^{2} \mathrm{D}_{5 / 2}$ & $4 p^{2} \mathrm{P}_{3 / 2}^{o}$ & \\
\hline 1301.800 & $\mathrm{Fe}$ VI & $4 s^{4} F_{9 / 2}$ & $4 p^{4} G_{9 / 2}^{o}$ & \\
\hline 1308.644 & $\mathrm{Fe}$ VI & $4 s^{4} F_{3 / 2}$ & $4 p^{4} G_{5 / 2}^{o}$ & \\
\hline 1309.910 & $\mathrm{Fe}$ VI & $4 s^{4} F_{7 / 2}$ & $4 p^{4} G_{7 / 2}^{o}$ & \\
\hline 1317.731 & $\mathrm{Fe}$ VI & $4 s^{4} F_{5 / 2}$ & $4 p^{4} G_{5 / 2}^{o}$ & \\
\hline 1324.286 & $\mathrm{Fe}$ VI & $4 s^{2} F_{5 / 2}$ & $4 p^{2} D_{3 / 2}^{o}$ & \\
\hline 1329.177 & $\mathrm{Fe}$ VI & $4 s^{2} F_{7 / 2}$ & $4 p^{2} D_{5 / 2}^{o}$ & \\
\hline 1330.971 & $\mathrm{Fe}$ VI & $4 s^{2} F_{5 / 2}$ & $4 p^{4} D_{5 / 2}^{o}$ & \\
\hline 1336.839 & $\mathrm{Fe}$ VI & $4 s^{4} P_{5 / 2}$ & $4 p^{2} \mathrm{P}_{3 / 2}^{\mathrm{o}}$ & \\
\hline 1337.692 & $\mathrm{Fe}$ VI & $4 s^{2} D_{5 / 2}^{3 / 2}$ & $4 p^{4} S_{3 / 2}^{o}$ & \\
\hline 1337.793 & $\mathrm{Fe}$ VI & $4 \mathrm{~s}^{2} \mathrm{~F}_{7 / 2}$ & $4 p^{4} D_{7 / 2}^{o}$ & \\
\hline 1361.817 & $\mathrm{Fe}$ VI & $4 \mathrm{~s}^{2} \mathrm{~F}_{5 / 2}$ & $4 p^{2} \mathrm{~F}_{5 / 2}^{o}$ & \\
\hline
\end{tabular}


Table A.5. Nickel lines identified in the HST/COS spectra of WD 0111+002 and PG 0109+111.

\begin{tabular}{|c|c|c|c|c|}
\hline \multirow{2}{*}{$\begin{array}{c}\text { Wavelength / } \\
\text { laboratory }\end{array}$} & \multirow[t]{2}{*}{ Ion } & \multicolumn{2}{|c|}{ Transition } & \multirow[t]{2}{*}{ Comment } \\
\hline & & low & up & \\
\hline 1357.064 & Ni IV & $4 s{ }^{6} D_{9 / 5}$ & $4 p^{6} \mathrm{P}_{712}^{0}$ & weak \\
\hline 1231.875 & $\mathrm{Niv}$ & $4 s^{3} D_{3}$ & $4 p^{3} \mathrm{P}_{2}^{\mathrm{o}}$ & weak \\
\hline 1232.524 & $\mathrm{Ni} \mathrm{V}$ & $4 s^{5} G_{4}$ & $4 p^{5} \mathrm{~F}_{3}^{\mathrm{o}}$ & weak \\
\hline 1232.807 & $\mathrm{Niv}$ & $4 \mathrm{~s}^{1} \mathrm{H}_{5}$ & $4 \mathrm{p}^{1} \mathrm{H}_{5}^{\mathrm{o}}$ & weak \\
\hline 1232.964 & $\mathrm{Niv}$ & $4 s^{3} D_{3}^{5}$ & $4 \mathrm{p}^{3} \mathrm{~F}_{4}^{\mathrm{o}}$ & weak \\
\hline 1233.257 & $\mathrm{Ni} \mathrm{V}$ & $4 s^{5} \mathrm{~F}_{5}$ & $4 \mathrm{p}^{5} \mathrm{~F}_{5}^{\mathrm{o}}$ & weak \\
\hline 1233.312 & $\mathrm{Ni} \mathrm{V}$ & $4 s^{5} G_{3}^{5}$ & $4 p^{5} \mathrm{~F}_{2}^{5}$ & weak \\
\hline 1235.831 & $\mathrm{Ni} \mathrm{V}$ & $4 \mathrm{~s}^{1} \mathrm{I}_{6}$ & $4 p^{5} G_{6}^{o}$ & weak \\
\hline 1242.043 & $\mathrm{Ni} \mathrm{V}$ & $4 \mathrm{~s}^{1} \mathrm{D}_{2}$ & $4 p^{3} G_{3}^{o}$ & \\
\hline $1244.174^{a}$ & $\mathrm{Ni} \mathrm{V}$ & $4 s^{7} S_{3}^{2}$ & $4 p^{7} \mathrm{P}_{4}^{3}$ & \\
\hline 1245.020 & $\mathrm{Niv}$ & $4 s^{1} F_{3}^{3}$ & $4 p^{3} \mathrm{~F}_{3}^{\mathrm{o}}$ & weak \\
\hline 1245.074 & $\mathrm{Ni} \mathrm{V}$ & $4 s^{3} G_{4}^{3}$ & $4 p^{3} F_{4}^{o}$ & weak \\
\hline 1245.203 & $\mathrm{Ni} \mathrm{V}$ & $4 s^{3} D_{2}$ & $4 \mathrm{p}^{3} \mathrm{~F}_{3}^{\mathrm{o}}$ & weak \\
\hline 1249.522 & $\mathrm{Niv}$ & $4 s^{5} \mathrm{G}_{5}$ & $4 p^{5} \mathrm{~F}_{4}^{3}$ & \\
\hline 1250.033 & $\mathrm{Ni} \mathrm{V}$ & $4 s^{5} G_{4}$ & $4 p^{5} \mathrm{~F}_{4}^{\mathrm{o}}$ & weak \\
\hline $1251.812^{a}$ & $\mathrm{Ni} \mathrm{V}$ & $4 s^{3} G_{5}^{4}$ & $4 p^{3} G_{5}^{0}$ & \\
\hline $1252.183^{a}$ & $\mathrm{Ni} \mathrm{V}$ & $4 s^{5} G_{6}$ & $4 p^{5} \mathrm{~F}_{5}^{\mathrm{o}}$ & \\
\hline 1253.980 & $\mathrm{Ni} \mathrm{V}$ & $4 s^{3} G_{3}$ & $4 \mathrm{p}^{3} \mathrm{G}_{3}^{\mathrm{o}}$ & \\
\hline $1257.626^{a}$ & $\mathrm{Niv}$ & $4 s^{5} G_{5}$ & $4 \mathrm{p}^{5} \mathrm{H}_{6}^{\mathrm{o}}$ & \\
\hline 1261.327 & $\mathrm{Ni} \mathrm{V}$ & $4 s^{3} F_{4}$ & $4 p^{3} G_{5}^{o}$ & \\
\hline 1261.760 & $\mathrm{Ni} \mathrm{V}$ & $4 s^{5} D_{4}^{4}$ & $4 p^{5} \mathrm{~F}_{5}^{\mathrm{o}}$ & \\
\hline $1264.501^{a}$ & $\mathrm{Ni} \mathrm{V}$ & $4 s^{7} S_{3}^{4}$ & $4 p^{7} \mathrm{P}_{3}^{0}$ & \\
\hline $1265.671^{b}$ & $\mathrm{Ni} \mathrm{V}$ & $4 \mathrm{~s}^{3} \mathrm{H}_{6}^{3}$ & $4 \mathrm{p}^{3} \mathrm{I}_{7}^{\mathrm{O}}$ & blend with unid. \\
\hline $1265.725^{b}$ & $\mathrm{Ni} \mathrm{V}$ & $4 s^{5} \mathrm{~F}_{3}^{0}$ & $4 p^{5} G_{4}^{o}$ & blend with unid. \\
\hline $1266.408^{a}$ & $\mathrm{NiV}$ & $4 s^{5} G_{4}$ & $4 \mathrm{p}^{5} \mathrm{H}_{5}^{\mathrm{o}}$ & \\
\hline 1270.677 & $\mathrm{Ni} \mathrm{V}$ & $4 \mathrm{~s}^{3} \mathrm{I}_{7}^{4}$ & $4 \mathrm{p}^{3} \mathrm{~K}_{8}^{\mathrm{o}}$ & \\
\hline 1273.204 & $\mathrm{Ni} \mathrm{V}$ & $4 s^{5} \mathrm{G}_{3}$ & $4 \mathrm{p}^{5} \mathrm{H}_{4}^{\mathrm{o}}$ & \\
\hline 1273.827 & $\mathrm{Ni}$ V & $4 \mathrm{~s}^{5} \mathrm{P}_{2}^{3}$ & $4 p^{5} \mathrm{P}_{3}^{0}$ & weak \\
\hline $1276.428^{a}$ & $\mathrm{Ni} \mathrm{V}$ & $4 s^{5} D_{3}^{2}$ & $4 \mathrm{p}^{5} \mathrm{~F}_{4}^{\mathrm{o}}$ & \\
\hline $1276.958^{a}$ & $\mathrm{Ni} \mathrm{V}$ & $4 s^{7} S_{3}{ }^{3}$ & $4 p^{7} P_{2}^{0^{0}}$ & \\
\hline 1279.325 & $\mathrm{Ni} \mathrm{V}$ & $4 s^{5} \mathrm{P}_{3}^{3}$ & $4 p^{5} D_{4}^{2}$ & weak \\
\hline 1279.720 & $\mathrm{Ni} \mathrm{V}$ & $4 s^{5} G_{2}^{3}$ & $4 \mathrm{p}^{5} \mathrm{H}_{3}^{\mathrm{o}}$ & \\
\hline 1280.138 & $\mathrm{Ni} \mathrm{V}$ & $4 \mathrm{~s}^{3} \mathrm{H}_{4}$ & $4 p^{3} I_{5}^{0}$ & weak \\
\hline 1282.201 & $\mathrm{Ni} \mathrm{V}$ & $4 \mathrm{~s}^{3} \mathrm{I}_{6}^{4}$ & $4 \mathrm{p}^{3} \mathrm{I}_{6}^{\mathrm{O}}$ & weak \\
\hline 1282.270 & $\mathrm{Ni} \mathrm{V}$ & $4 \mathrm{~s}^{3} \mathrm{I}_{5}$ & $4 p^{3} I_{6}^{o}$ & weak \\
\hline 1282.724 & $\mathrm{Ni} \mathrm{V}$ & $4 \mathrm{~s}^{3} \mathrm{I}_{7}$ & $4 p^{3} I_{6}^{0}$ & weak \\
\hline 1287.553 & $\mathrm{Ni} \mathrm{V}$ & $4 s^{3} D_{3}$ & $4 \mathrm{p}^{3} \mathrm{~F}_{4}^{\mathrm{o}}$ & weak \\
\hline 1287.628 & $\mathrm{Ni} \mathrm{V}$ & $4 s^{1} D_{2}^{3}$ & $4 p^{1} \mathrm{~F}_{3}^{\mathrm{o}}$ & weak \\
\hline 1287.808 & $\mathrm{Ni} \mathrm{V}$ & $4 s^{5} D_{1}^{2}$ & $4 \mathrm{p}^{5} \mathrm{~F}_{2}^{\mathrm{o}}$ & weak \\
\hline 1300.979 & $\mathrm{Ni} \mathrm{V}$ & $4 s^{5} S_{2}^{1}$ & $4 p^{5} \mathrm{P}_{1}^{\mathrm{o}}$ & \\
\hline 1304.870 & $\mathrm{Ni} \mathrm{V}$ & $4 s^{3} I_{5}^{2}$ & $4 p^{3} I_{5}^{0}$ & \\
\hline 1305.696 & $\mathrm{Ni} \mathrm{V}$ & $4 s^{3} I_{6}$ & $4 \mathrm{p}^{3} \mathrm{~K}_{7}^{\mathrm{o}}$ & weak \\
\hline 1306.238 & $\mathrm{Ni} \mathrm{V}$ & $4 \mathrm{~s}^{3} \mathrm{I}_{7}^{0}$ & $4 \mathrm{p}^{3} \mathrm{~K}_{7}^{\mathrm{o}}$ & weak \\
\hline 1306.624 & $\mathrm{Ni} \mathrm{V}$ & $4 s^{5} G_{6}$ & $4 p^{5} \mathrm{G}_{6}^{\mathrm{o}}$ & \\
\hline 1307.603 & $\mathrm{NiV}$ & $4 s^{5} S_{2}^{6}$ & $4 p^{5} \mathrm{P}_{2}^{0}$ & \\
\hline 1311.106 & $\mathrm{Ni} \mathrm{V}$ & $4 s^{5} G_{5}^{2}$ & $4 p^{5} G_{5}^{2}$ & \\
\hline $1312.718^{a}$ & $\mathrm{Ni} \mathrm{V}$ & $4 \mathrm{~s}^{3} \mathrm{I}_{5}$ & $4 p^{3} K_{6}^{0}$ & \\
\hline 1313.280 & $\mathrm{Ni} \mathrm{V}$ & $4 s^{5} G_{4}$ & $4 p^{5} G_{4}^{o}$ & \\
\hline $1314.330^{a}$ & $\mathrm{Ni} \mathrm{V}$ & $4 s^{5} G_{3}^{4}$ & $4 p^{5} G_{3}^{0}$ & \\
\hline 1314.349 & $\mathrm{Ni} \mathrm{V}$ & $4 \mathrm{~s}^{1} \mathrm{H}_{5}$ & $4 \mathrm{p}^{1} \mathrm{I}_{6}^{\mathrm{O}}$ & \\
\hline 1314.682 & $\mathrm{Ni} \mathrm{V}$ & $4 s^{5} \mathrm{G}_{2}$ & $4 \mathrm{p}^{5} \mathrm{G}_{2}^{0}$ & weak \\
\hline 1317.447 & $\mathrm{Ni} \mathrm{V}$ & $4 \mathrm{~s}^{1} \mathrm{I}_{6}$ & $4 \mathrm{p}^{1} \mathrm{~K}_{7}^{\mathrm{o}}$ & \\
\hline 1318.327 & $\mathrm{Ni} \mathrm{V}$ & $4 s^{3} D_{2}$ & $4 \mathrm{p}^{3} \mathrm{~F}_{3}^{\mathrm{o}}$ & weak \\
\hline $1318.515^{a}$ & $\mathrm{Ni} \mathrm{V}$ & $4 s^{5} S_{2}^{2}$ & $4 \mathrm{p}^{5} \mathrm{P}_{3}^{\mathrm{o}}$ & \\
\hline 1323.562 & $\mathrm{Ni} \mathrm{V}$ & $4 \mathrm{~s}^{3} \mathrm{H}_{6}$ & $4 \mathrm{p}^{3} \mathrm{H}_{6}^{\mathrm{o}}$ & weak \\
\hline 1323.977 & $\mathrm{Ni} \mathrm{V}$ & $4 s^{3} \mathrm{G}_{3}$ & $4 \mathrm{p}^{3} \mathrm{H}_{4}^{\mathrm{o}}$ & \\
\hline $1329.358^{a}$ & $\mathrm{Ni} \mathrm{V}$ & $4 s^{3} G_{4}$ & $4 \mathrm{p}^{3} \mathrm{H}_{5}^{\mathrm{O}}$ & \\
\hline $1336.136^{a}$ & $\mathrm{Ni} \mathrm{V}$ & $4 s^{3} G_{5}^{4}$ & $4 \mathrm{p}^{3} \mathrm{H}_{6}^{\mathrm{o}}$ & \\
\hline 1347.720 & Ni V & $4 s^{3} G_{4}$ & $4 p^{3} \mathrm{~F}_{3}^{\mathrm{o}}$ & weak \\
\hline
\end{tabular}

Notes. ${ }^{(a)}$ Also seen in PG 0109+111. ${ }^{(b)}$ Only seen in PG 0109+111.

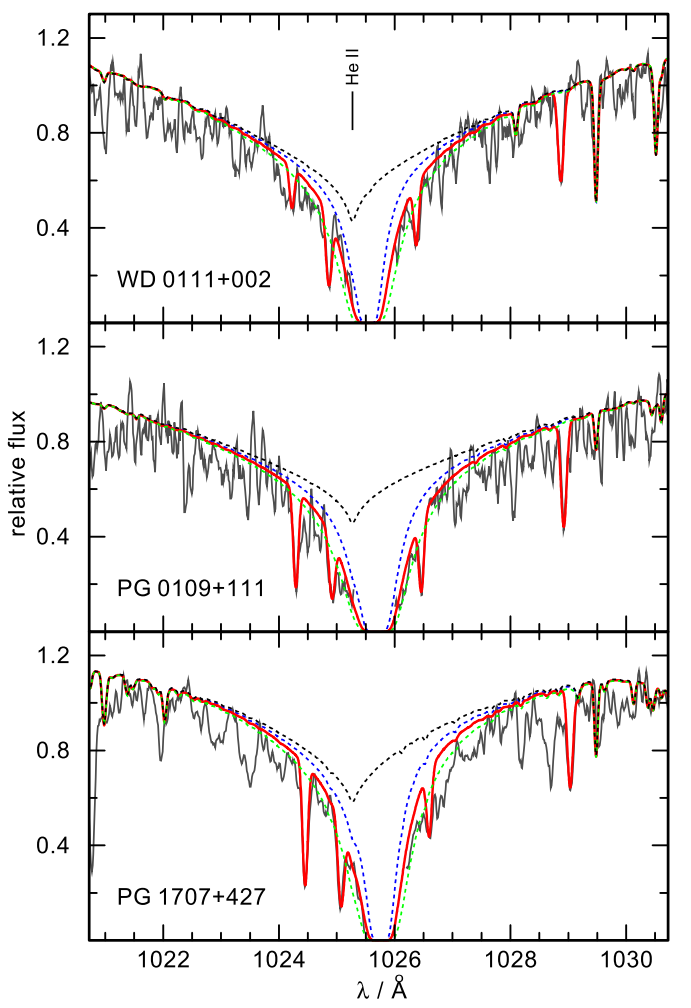

Fig. A.1. Synthetic spectra around Ly $\beta$ from our final models (black, dashed graphs) for the program stars compared with the FUSE observations (black). Red graphs: interstellar absorption with neutral hydrogen column densities as given in Sect.3.2 applied. The adjacent dashed graphs indicate the error limits.

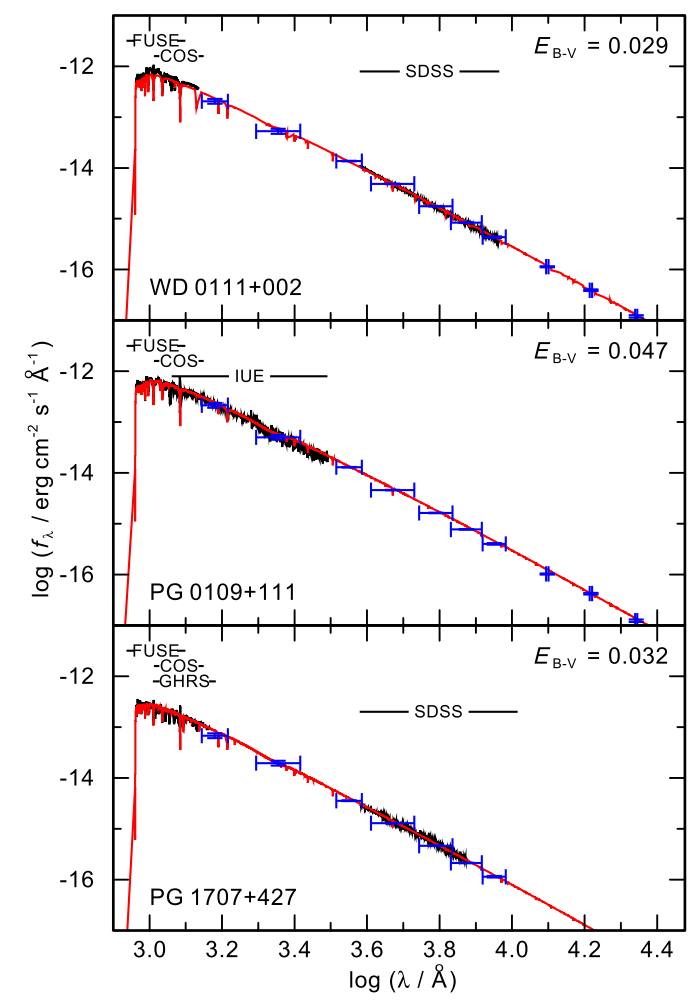

Fig. A.2. Spectral energy distribution of our program stars compared to the final, reddened models (red graphs). Models of WD 0111+002, and PG 0109+111 were normalized to the 2MASS $\mathrm{H}$ magnitude and that of PG $1707+427$ to the SDSS z magnitude. Magnitudes from GALEX, SDSS, and 2MASS are plotted in blue with their error bars. 


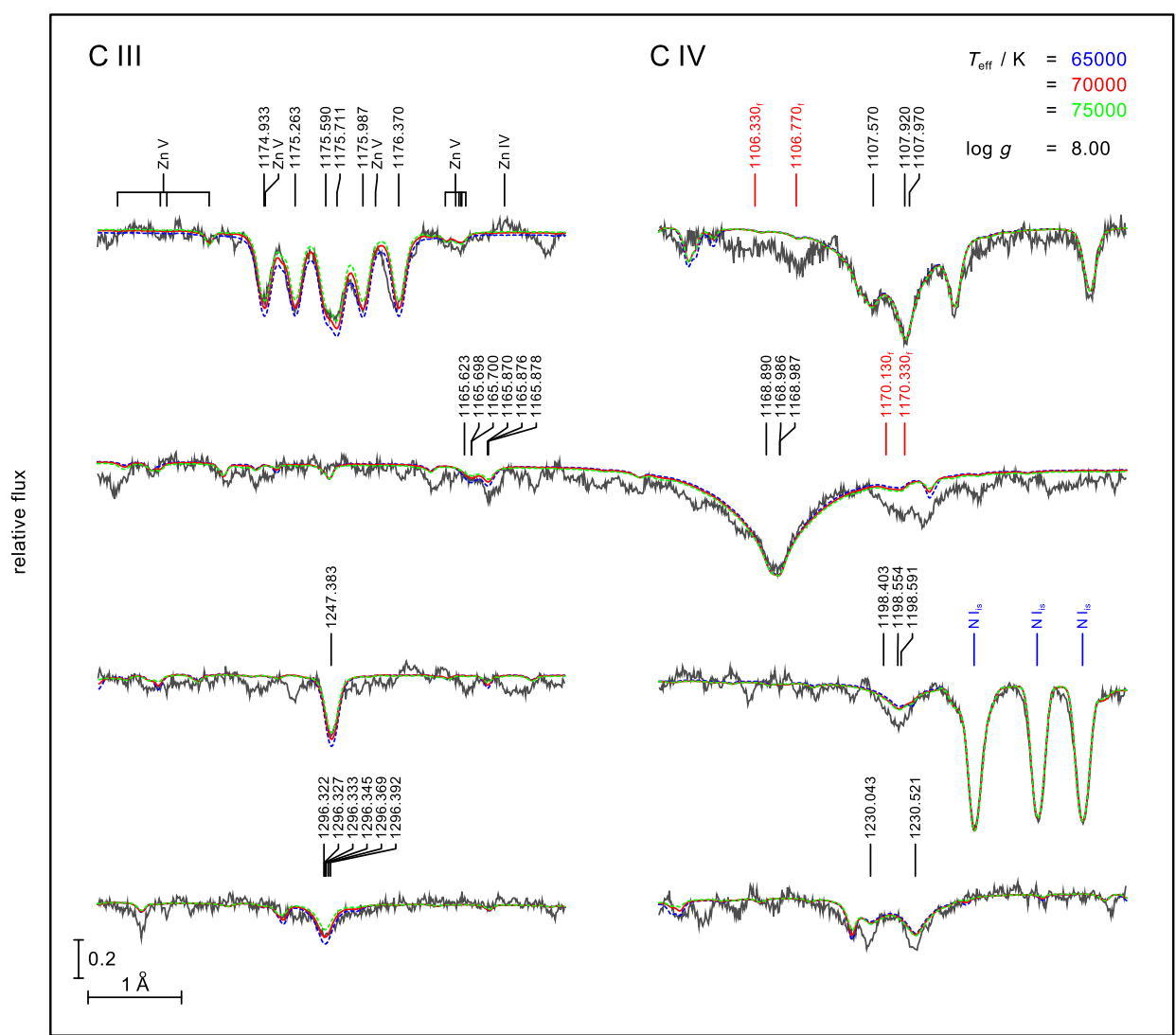

$\Delta \lambda / \AA$

Fig. A.3. Like Fig. 2, but for PG 0109+111.

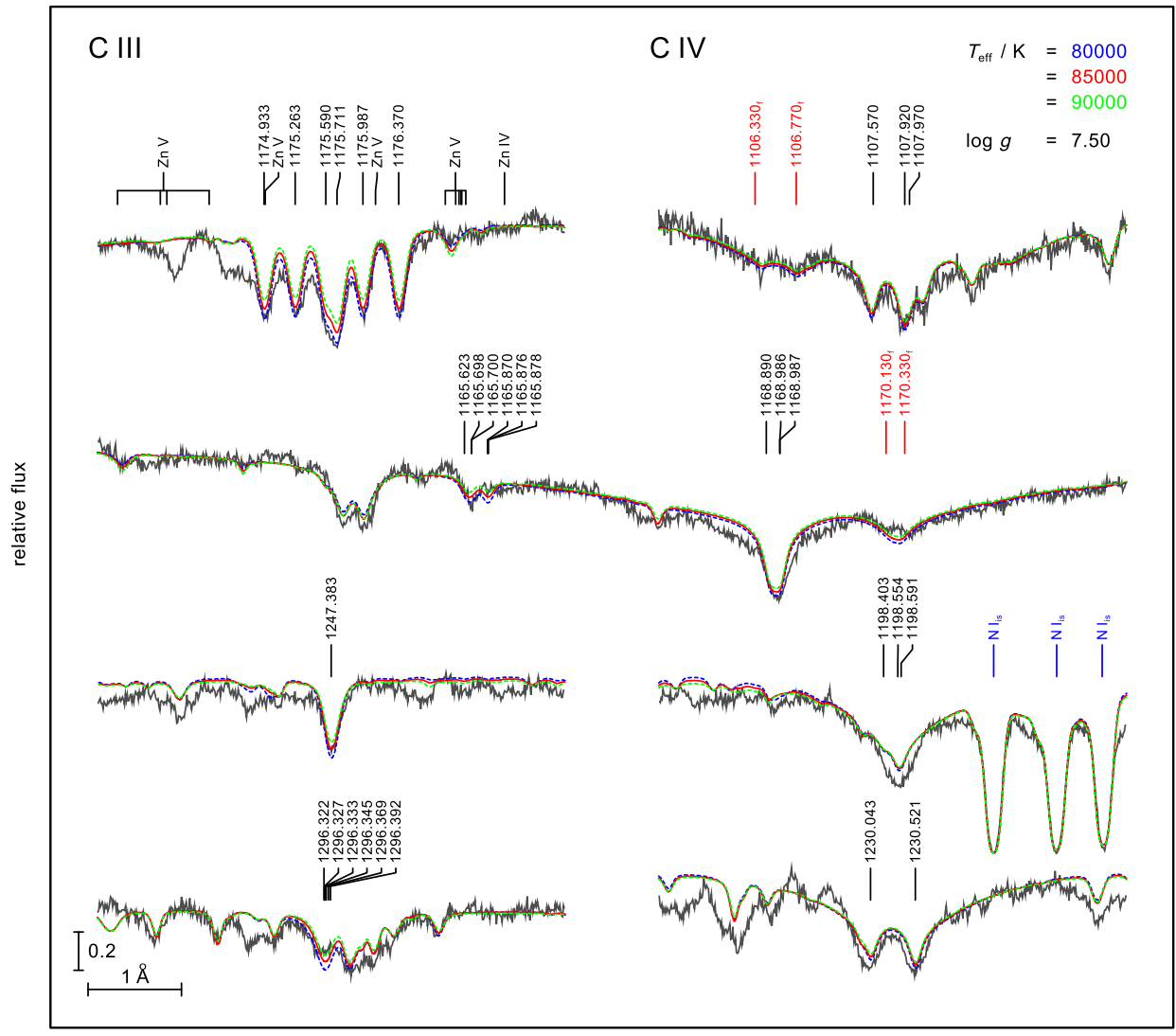

$\Delta \lambda / \AA$

Fig. A.4. Like Fig. 2, but for PG 1707+427. 
D. Hoyer et al.: Search for trans-iron elements in hot, helium-rich white dwarfs with HST/COS

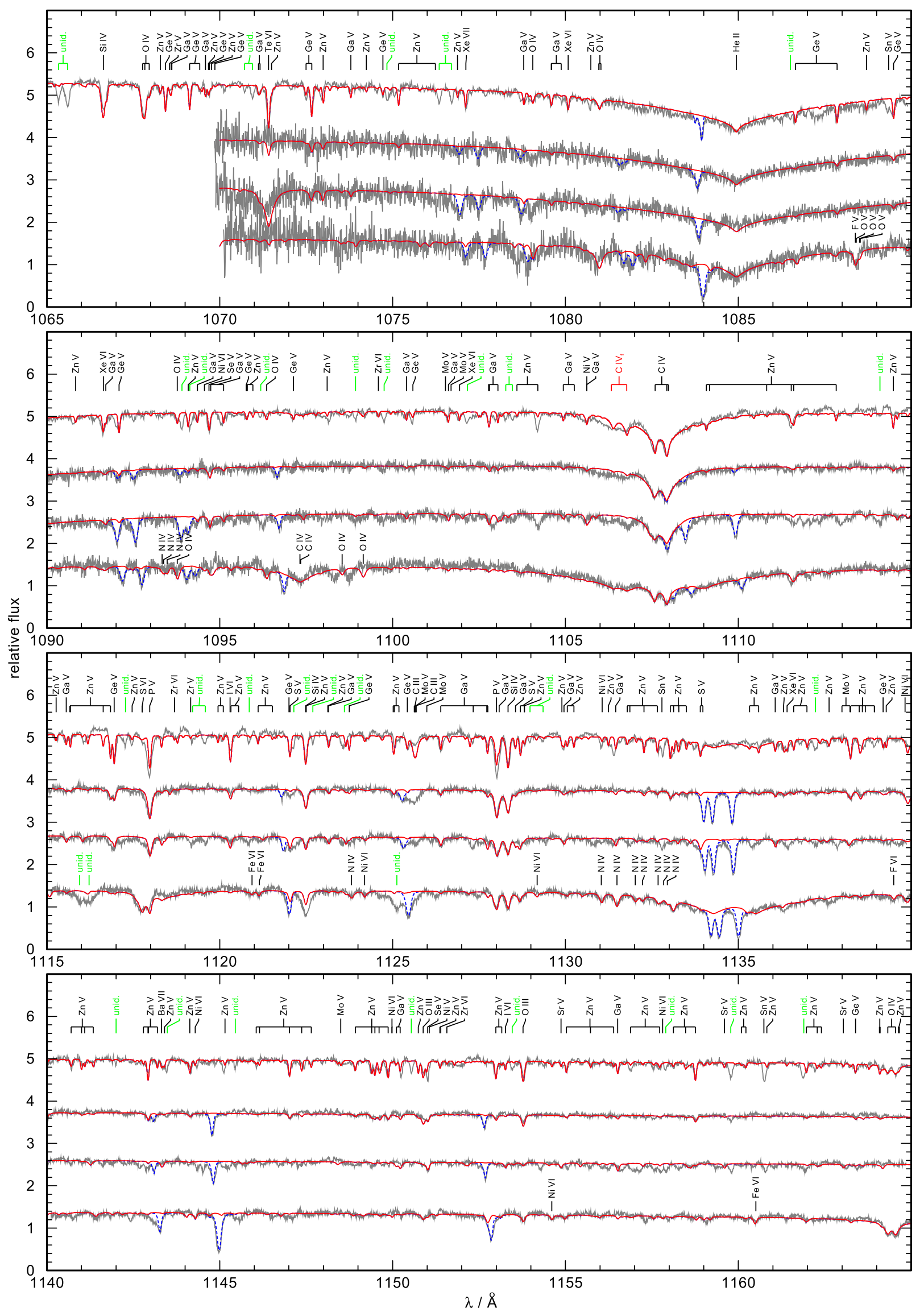

Fig. A.5. Complete HST/COS spectra of our three program stars (three bottom spectra in each panel) together with the HST/STIS spectrum of the comparison DO star RE 0503-289 (top spectrum). Overplotted (red graphs) are our final models and the final model for RE 0503-289 from Rauch et al. (2017b). Blue dashed graphs indicate ISM lines. 


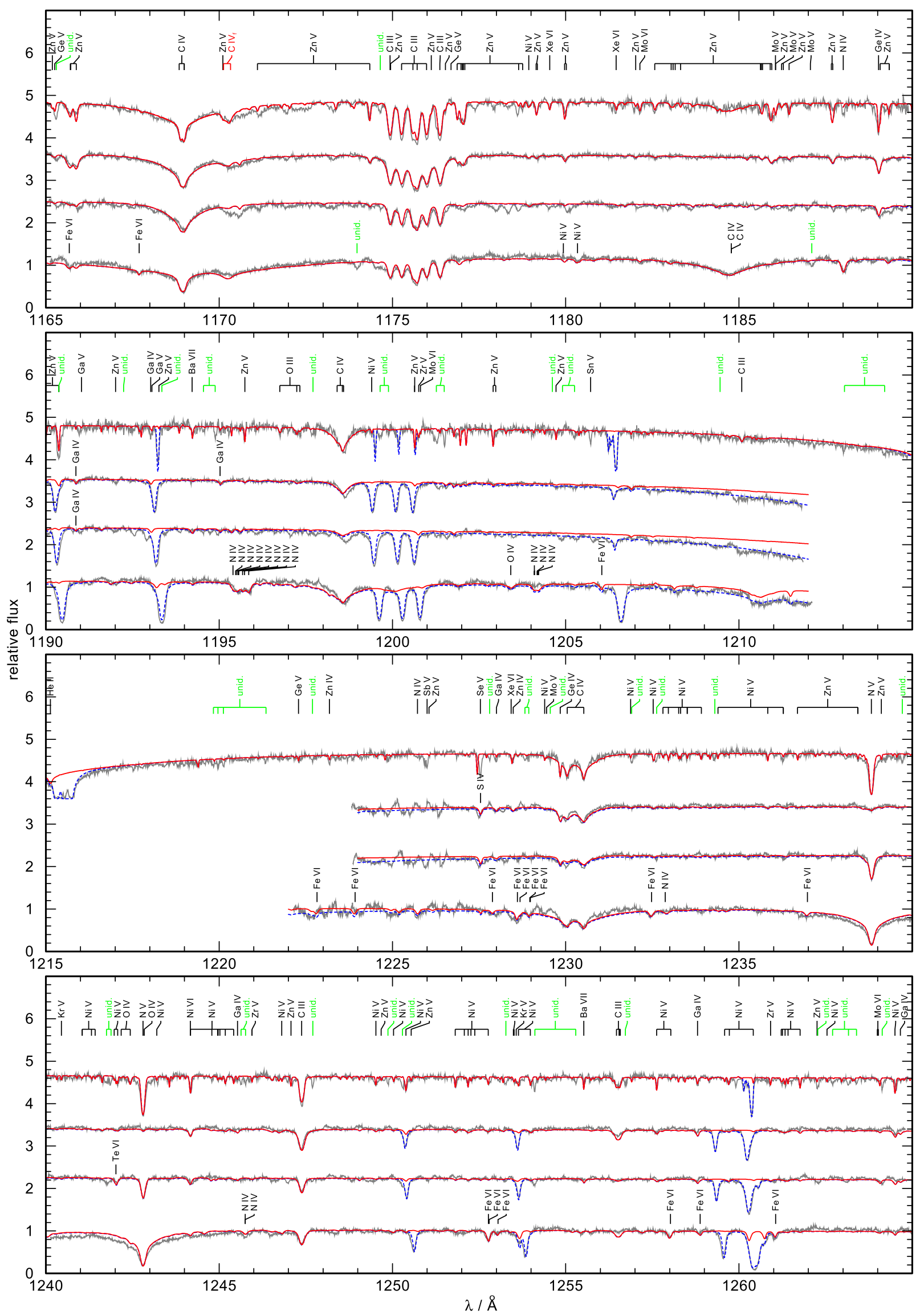

Fig. A.5. continued. 
D. Hoyer et al.: Search for trans-iron elements in hot, helium-rich white dwarfs with HST/COS

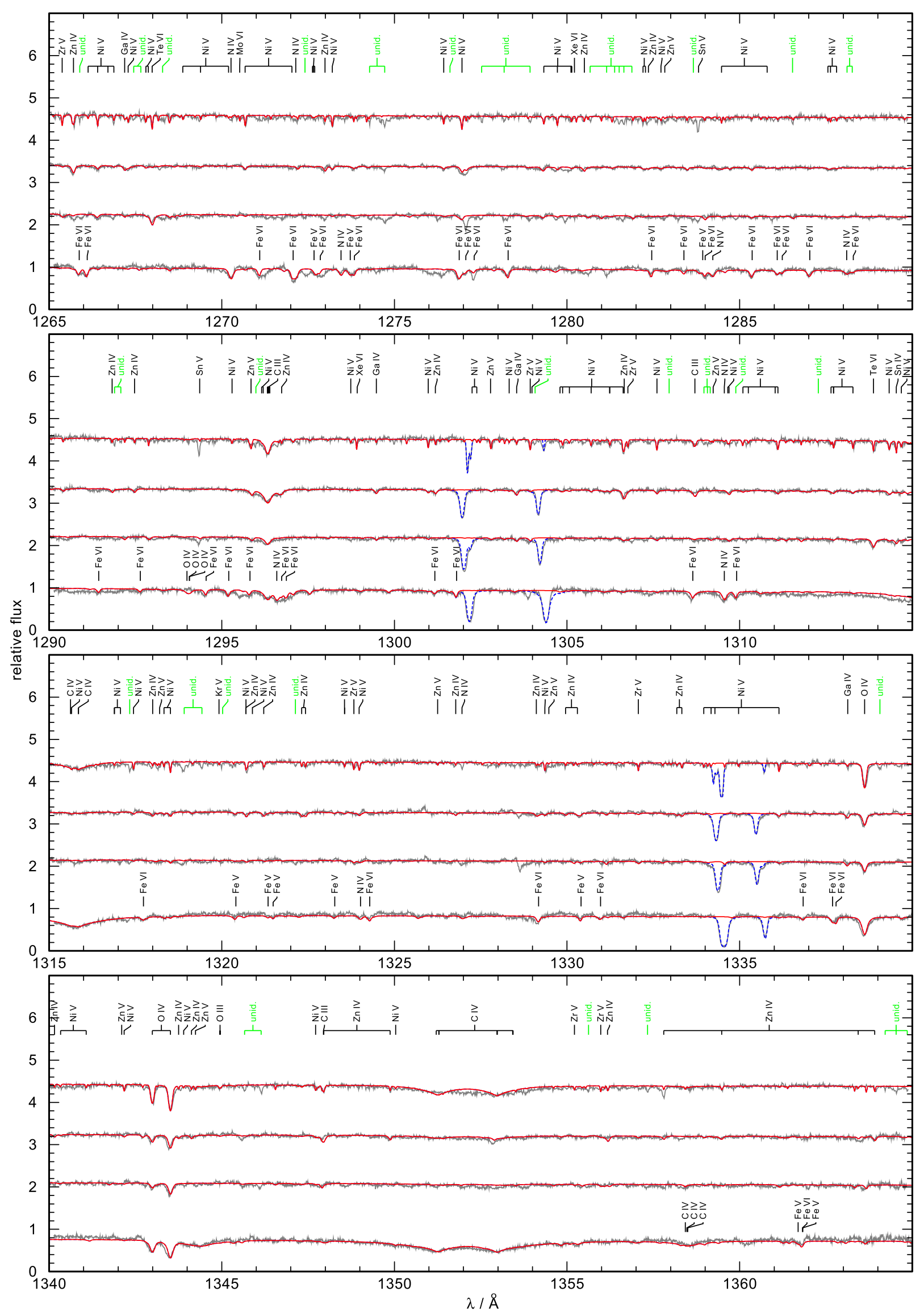

Fig. A.5. continued. 